

\section{polisemie}

\section{I}

\section{1}

Direzione

Stefano Milonia (University of Warwick)

Comitato scientifico

Giulia Bassi (Università degli Studi di Siena)

Mario Cianfoni (Sapienza Università di Roma)

Stefano Colangelo (Alma Mater Studiorum - Università degli Studi di Bologna)

Luigi Marinelli (Sapienza Università di Roma)

Carlo Pulsoni (Università degli Studi di Perugia)

Niccolò Scaffai (Università degli Studi di Siena)

Costantino Turchi (Sapienza Università di Roma)

Fabio Zinelli (École Pratique des Hautes Études)

\section{Redazione}

Stefano Bottero (Università Ca' Foscari Venezia)

Mattia Caponi (Sapienza Università di Roma)

Carlo Londero (Università degli Studi di Udine)

Giorgio Tranchida (Alma Mater Studiorum - Università degli Studi di Bologna)

Samuele Maria Visalli (Sapienza Università di Roma)

Arianna Saggio (Sapienza Università di Roma)

Andrea Bongiorno (Aix-Marseille Université)

Giulia Boitani (University of Cambridge)

Alessandra Frustaci

Polisemie è una rivista annuale pubblicata dalla University of Warwick Press.

Gli articoli pubblicati nella sezione Saggi sono sottoposti a double-blind peer review.

Licenza Creative Commons - Attribuzione (CC-BY 4.0).

ISSN: 2634-1867

DOI: 10.31273 /polisemie.v1

Immagine copertina: Serse Luigetti 


\title{
LE POESIE ITALIANE DI QUESTI ANNI (2005-2020)
}

\author{
Claudia Crocco
}

\section{Premesse}

Il titolo di questo intervento non è originale, bensì riprende quello - ben più autorevole - di Franco Fortini (1953) pubblicato poi in Saggi ed epigrammi (2003). Ho già ripreso questo titolo (e questo spunto) in un intervento del 2014 pubblicato sull'«Ulisse», del quale questo articolo vuole essere una ideale continuazione, ${ }^{1}$ ma con una differenza. Mentre nel 2014 ho seguito lo schema di Fortini, presentando dei brevi ritratti di pochi autori esemplari, nelle pagine che seguono si procederà diversamente: sono stati considerati molti più libri e autori, dei quali ci si servirà per parlare di tendenze più generali nella poesia contemporanea italiana.

La seconda premessa da fare riguarda la periodizzazione: esiste una poesia degli anni Duemila, distinta da quella dei decenni precedenti? E quali sono le sue caratteristiche distintive? Ancora oggi, nelle antologie e negli studi di ampio respiro sulla poesia contemporanea, un punto di riferimento è il saggio introduttivo di Berardinelli a Il pubblico della poesia del 1975. Spesso si rimanda a Effetti di deriva per lamentare che la poesia di oggi è ancora più dispersiva di allora, e ancora più separata da quella della generazione che l'ha preceduta - cioè gli antologizzati da Berardinelli -, come conseguenza dell'ulteriore moltiplicazione degli autori e dell'accentuarsi dei fenomeni di cordate fra poeti e critici, figure che troppo spesso coincidono. ${ }^{2}$ Nelle pagine che seguono non ci si preoccuperà di contestare le tesi sulla fine della poesia - un topos critico con il quale molti ancora sentono di doversi

\footnotetext{
${ }^{1}$ Alfonso Berardinelli, Effetti di deriva, in Alfonso Berardinelli, Franco Cordelli, a cura di, Il pubblico della poesia, Cosenza, Lerici, 1975, pp. 7-29.

${ }^{2}$ E un'idea ancora presente, ad esempio, in Parola plurale, cioè l'ultima antologia d'autore della poesia contemporanea italiana: cfr. Deriva di effetti in Giancarlo Alfano, Alessandro Baldacci, Cecilia Bello Minciacchi, Andrea Cortellessa, Massimiliano Manganelli, Raffaella Scarpa, Fabio Zinelli, Paolo Zublena, Parola plurale. Sessantaquattro poeti fra due secoli, Roma, Sossella, 2005, pp. 7-29, soprattutto pp. 8-10.
} 
confrontare, quando iniziano a scrivere, che in questa sede verrà considerato superato. La vera domanda, nel 2021, è un'altra: quanto sono cambiate le cose, rispetto al 1975?

In queste pagine si adotterà una prospettiva abbastanza continuista, cioè basata sull'idea che la disgregazione del campo e il cambiamento nel rapporto con la tradizione iniziati negli anni Settanta caratterizzino anche la poesia italiana contemporanea degli anni Duemila. Alcuni dei fenomeni descritti da Berardinelli nel 1975 sono ancora attuali, soprattutto da un punto di vista sociologico; tuttavia va riconosciuto che il passaggio alla rete ha modificato il pubblico della poesia. ${ }^{3}$ Larrivo di internet ha accelerato il processo di sovrapposizione fra autori e pubblico, causando la quasi totale scomparsa del secondo; ${ }^{4}$ ha inciso sia sui luoghi del dibattito (dalle riviste cartacee ai blog, dalle riviste online ai social network), sia sui modi (presenza minore di filtri determinati dalle competenze, affermazione della logica dello user generated content, ecc.), sia sulle sedi di pubblicazione - e, dunque, sui centri di attribuzione di capitale simbolico. Eppure, se le cose sono cambiate in modo significativo nei luoghi in cui si parla di poesia, forse la realtà è più sfumata per quanto riguarda il rapporto tra poesia ed editoria. Per molti poeti di oggi è importante pubblicare su alcuni blog, essere conosciuti online, avere un profilo su qualche social network; ma tutto ciò non ha davvero scavalcato la pubblicazione di un libro cartaceo. Al contrario: i libri cartacei circolano di più $\mathrm{e}$ in maniera più mirata (per quanto con numeri sempre risibili) da quando social network e blog permettono di raggiungere in maniera immediata un pubblico selezionato e già interessato, nonché di attuare strategie di marketing a un costo relativamente basso. Lo scenario editoriale è cambiato negli ultimi anni, ma le

\footnotetext{
${ }^{3}$ Svariati studi sull'argomento sono già usciti, e probabilmente altri verranno scritti e pubblicati nei prossimi anni. Per quanto riguarda il contesto italiano, cfr. in particolare Francesco Guglieri, Michele Sisto, Verifica dei poteri 2.0. Critica e militanza letteraria in internet (1999-2009), in «Allegoria», 61, 2010, pp. 153-174; Gherardo Bortolotti, Oltre il pubblico: la letteratura e il passaggio alla rete, in Idee della prosa, a cura di Gilda Policastro, numero monografico di «Nuova prosa», 6, 2014, pp. 77-146, poi pubblicato online su Le parole e le cose, 22 dicembre 2014 (si cita dalla versione online); Andrea Lombardi, L'esperienza di «Nazione indiana» nella storia del web letterario italiano, in «L'Ulisse», 19, 2016, pp. 47-63; Paolo Giovannetti, La poesia italiana degli anni Duemila. Un percorso di lettura, Roma, Carocci, 2017, pp. 95-115; Guido Mazzoni, Sulla storia sociale della poesia contemporanea in Italia, in «Ticontre. Teoria Testo Traduzione», VIII, 2017, pp. 1-26.

${ }^{4}$ «Soprattutto quel soggetto più o meno fantasmatico e ipostatizzato che era il pubblico (declinato eventualmente, secondo i dibattiti e le posizioni, anche come popolo, nazione o gente) e sulla cui presenza più o meno muta si è costruita una parte non secondaria delle nozioni moderne di autore, di opera d'arte e di cultura, si dissolve in un pulviscolo autoriale, in una specie di insorgenza mediatica diffusa, più partecipativa che creativa ma comunque gestita in prima persona, attivamente e non in termini di mera ricezione» (Bortolotti, Oltre il pubblico).
} 
novità hanno riguardato soprattutto il ruolo delle collane.

Fino agli anni Novanta le più importanti collane di poesia italiana erano quelle delle grandi case editrici - Mondadori ed Einaudi, innanzitutto -, mentre la situazione oggi è molto diversa: le collane più importanti nella storia della poesia del Novecento hanno smesso di promuovere nuove voci, limitandosi a pubblicare un gruppo ristretto di poeti già affermati - seppure con qualche eccezione.

Lo Specchio di Mondadori, dopo una fase di pochissime uscite negli anni Ottanta e Novanta, ${ }^{5}$ all'inizio dei Duemila - sotto la direzione di Cucchi e Riccardi - ha ripreso a pubblicare in modo più regolare, consolidando un piccolo canone di autori nati negli anni Cinquanta (Milo De Angelis, Antonella Anedda, Maurizio Cucchi, Biancamaria Frabotta), ma ha smesso di svolgere la funzione propulsiva e di scoperta che aveva avuto in passato; i pochi poeti delle generazioni successive accolti nello Specchio sembrano scelti in modo quasi accidentale. Sul piano internazionale, lo Specchio ha continuato a pubblicare poesia straniera, ma con scelte meno coraggiose che in passato, rilanciando soprattutto autori già affermati (Seamus Heaney, Adonis, Jorie Graham). Anche Einaudi ha continuato a diffondere alcuni nomi già famosi (Valerio Magrelli, Patrizia Cavalli, Alda Merini) e ne ha accolti pochi fra i più giovani; inoltre ha pubblicato autori già noti in quanto artisti in campi diversi da quello poetico (ad esempio romanzieri, come Aldo Nove e Andrea Bajani), che hanno potuto garantire un pubblico e un ritorno economico più ampio di quello della poesia. Mentre le due principali collane di poesia italiana di fine Novecento perdevano capacità propulsiva e canonizzante, si consolidava il potere delle case editrici medie (Donzelli, Transeuropa, Aragno, Interlinea) e l'aspirante avanguardia degli anni Duemila concentrava le proprie pubblicazioni in piccole o piccolissime $-{ }^{6}$ ma il discorso merita di essere approfondito altrove.

Per concludere questo punto, anche sul piano formale non c'è una separatezza totale nei libri di chi esordisce negli anni Dieci rispetto agli autori esordienti uno, due o tre decenni prima. Va segnalato, piuttosto, che gli esordienti della generazione del Pubblico della poesia, in alcuni casi, hanno influenzato ed influenzano in modo sostanziale i poeti più giovani. De Angelis è il caso più

${ }^{5}$ Cfr. Giulia Iannuzzi, Lo Specchio, in Gian Carlo Ferretti, Giulia Iannuzzi, Storie di uomini e libri. L'editoria italiana attraverso le sue collane, Roma, minimum fax, 2014, pp. 88-96, soprattutto pp. 94-96. Cfr. anche Francesca Sante, I grandi editori della poesia, in Design Thinking: studio preparatorio per un progetto in versi, tesi di laurea, Corso di Laurea in Economia e Gestione delle Arti e Attività culturali, Università Ca' Foscari Venezia, a. 2017/2018, relatore prof.ssa Monica Calcagno, controrelatore prof.ssa Ricciarda Ricorda, pp. 40-42.

${ }^{6}$ Ad esempio La Camera Verde, Tielleci, Benway Series, Tic, Prufrock. Sulle strategie editoriali del gruppo di Prosa in prosa e GAMMM, e a proposito del loro rapporto con l'avanguardia, cfr. Claudia Crocco, La poesia in prosa in Italia. Dal Novecento a oggi, Roma, Carocci, 2021, pp. 11-18 e pp. 167173. 
evidente, ma non certo l'unico. ${ }^{7}$ Alcuni di quelli che un tempo erano autori 'nuovi', apparentemente fuori da ogni canone e indecifrabili sia su un piano formale sia per postura autoriale, sono diventati i "nuovi maestri" di oggi, e l'editoria ha contribuito a questo fenomeno. Non solo: molti poeti di quella generazione si sono adeguati alle nuove strategie comunicative della poesia del ventunesimo secolo, che sfruttano come o più dei giovani. Sono pochi, ad esempio, quelli che non si servono dei social network. Fra le generazioni, insomma, si notano sia fenomeni di continuità sia altri di rottura.

Passiamo, quindi, alle tendenze riscontrabili nei testi. Ci si limiterà ad alcune osservazioni preliminari, che non hanno la pretesa di esaurire il discorso. Vale la pena di precisare che in queste pagine non ci si propone una ricostruzione del campo indipendente da questioni di gusto - come si farebbe in sede antologica -, bensì una panoramica della poesia italiana degli anni 2005-2020 che appare meritevole a chi scrive: cioè quella che propone una visione modernista della realtà. In altre parole, verrà considerata la poesia che dà una rappresentazione dell'interiorità e dei rapporti umani nel mondo contemporaneo (già questo pone un limite significativo al corpus). Rimangono esclusi da questo quadro i libri di poesia che parlano di poesia o di letteratura, nei quali la forma o la citazione è l'obiettivo stesso dell'azione poetica oppure una forma di divertissement, nonché la poesia esclusivamente performativa.

Chiariti i criteri dei quali si è tenuto conto nella scelta del corpus, è possibile individuare cinque tendenze all'interno della poesia contemporanea italiana. La prima e la seconda hanno a che fare con il punto di vista di chi prende la parola nel testo e con il tipo di soggettività rappresentata, la terza è definita a partire da una caratteristica formale dei testi (la scelta del poemetto), che però ne condiziona anche la struttura enunciativa; la quarta e la quinta riguardano caratteristiche formali. Per questioni di spazio, in queste pagine si approfondiranno soltanto i primi tre orientamenti; si rimanda a un lavoro futuro una analisi più dettagliata degli ultimi due, Ibridi e Prosa, che saranno in questa sede solamente accennati.

\footnotetext{
${ }^{7}$ Sull'influenza di De Angelis, cfr. almeno Andrea Afribo, Deangelisiana, in Poesia '70-'80: le nuove generazioni. Geografia e storia, opere e percorsi, letture e commento, selezione di contenuti dal convegno (Torino, 15-17 dicembre 2015), a cura di Davide Dalmas, Stefano Giovannuzzi, Beatrice Manetti e Sabrina Stroppa, Genova, San Marco dei Giustiniani, pp. 113-132; Damiano Sinfonico, Scuola deangelisiana: l'esempio della collana Niebo, in «Ticontre. Teoria Testo Traduzione», VIII, 2017, pp. 73-85.
} 


\section{Voci}

La prima tendenza è quella a rappresentare quadri narrativi nei quali personaggi diversi dall'io prendono la parola, oppure con apparente assenza di voicing. ${ }^{8}$ Questo primo gruppo è molto variegato, sia perché comprende testi in versi e (la maggioranza, in realtà) testi in prosa, sia perché è trasversale a numerosi autori: il nucleo principale è costituito dai alcuni libri già considerati nell'intervento del 2014 che fa da premessa a questo articolo, ${ }^{9}$ ad esempio I mondi di Guido Mazzoni (Roma, Donzelli, 2010), ai quali si aggiunge ora La pura superficie (Roma, Donzelli, 2017); Tecniche di basso livello (Sant'Angelo in Formis, Lavieri, 2009) e Senza paragone (Massa, Transeuropa, 2013) di Gherardo Bortolotti (entrambi confluiti, insieme a Quando arrivarono gli alieni. Parti 234-361, Colorno, Tielleci, 2016, nella trilogia Low, Roma, Tic, 2020); Avventure minime (Massa, Transeuropa, 2014) di Alessandro Broggi (con il recente Noi, Roma, Tic, 2021); le opere di Anedda (soprattutto con il Catalogo della gioia e Dal balcone del corpo, pubblicati per Mondadori nel 2003 e nel 2007, ai quali si aggiungono ora Historiae, Torino, Einaudi, 2018, e Geografie, Milano, Garzanti, 2021). Vanno considerati, quindi, i libri di Paolo Maccari, con Fermate (Roma, Elliot, 2017) e l'autoantologia Iferri corti (Faloppio, Lietocolle, 2020), Primine di Alessandra Carnaroli (Milano, Edizioni del Verri, 2017) le prose di Esercizi di vita pratica di Gilda Policastro (Costa di Rovigo, Prufrock, 2017); infine, i libri di autori più giovani come Jacopo Ramonda (Una lunghissima rincorsa, Roma, Bel Ami, 2014, e poi Omonimia, Interlinea, 2019), Carmen Gallo (Le fuggitive, Torino, Aragno, 2020), Simone Burratti (Progetto per S., Varese, Nuova Editrice Magenta, 2017), ma anche Antonio Lanza e Valentino Ronchi - su questi ultimi due ci si soffermerà più

\footnotetext{
${ }^{8}$ Prendo in prestito questa parola da Theory of the Lyric di Culler, ma le attribuisco un significato lievemente diverso: «Ever since the Greeks, lyrics have generally been encountered as writing that readers articulate, silently or orally, but the importance of sound for lyric has made the notion of voice loom large, both in poems themselves and in the critical tradition. The fact that written lyrics persist in alluding to lyres and presenting themselves as sung long after they take the form of writing emphasizes the importance of their aural dimension for their characteristic effect [...]. That is to say, to read something as lyric is allegedly to lend phenomenal form to something like a voice, to convince ourselves that we are hearing a voice [...]. Rather than imagine that lyrics embody voices, we do better to say that they create effects of voicing, of aurality» (Jonathan Culler, Theory of the Lyric, Cambridge, Harvard University Press, 2015, pp. 34-35). Mentre Culler intende il voicing come una sorta di residuo orale (e ritmico) di una voce, in queste pagine si presterà attenzione alla presenza di una voce intesa come presenza di un soggetto enunciativo. La categoria di soggetto enunciativo è tratta da Käte Hamburger, La logica della letteratura, a cura di Eleonora Caramelli, Bologna, Pendragon, 2015, pp. 303-309.

${ }^{9} \mathrm{Si}$ è scelto di considerare soprattutto gli autori non presenti nell'articolo del $2014 \mathrm{o}$, in pochi casi, i libri che i poeti già affrontati nel 2014 hanno pubblicato dopo quella data.
} 
avanti. Si tratta di un gruppo intergenerazionale e soprattutto con molte sfumature interne, sia tematiche che stilistiche. Ciò che questi testi hanno in comune è il fatto di sfidare una caratteristica solitamente considerata intrinseca alla poesia, ossia il monologismo. ${ }^{10}$ Per comprendere le strategie attraverso cui una parte della poesia italiana tenta di accedere a soggettività diverse da quella dell'autore, consideriamo qualche esempio.

C’è un versante, in questo gruppo, che tende al grottesco, dunque a una rappresentazione della realtà paradossale e - a una lettura superficiale - persino comica, che in realtà ne mette a fuoco i punti di contraddizione: è il caso di alcuni testi di Broggi e, fra gli esordienti dell'ultimo decennio, di Burratti:

Prezzemolo, salvia, rosmarino e timo: la magia è un'arte che può soddisfare tutte le esigenze. Rianimare amori spenti, spezzare legami che non volete più, allontanare una persona. Avvolti nel mistero e intrisi di leggenda, i filtri d'amore sono stati presenti nella storia umana per secoli. Ieri come oggi moltissime persone chiedono filtri e pozioni per conquistare il cuore della persona amata. Anziché consultare un medico o uno psicoterapeuta, decidono di rivolgersi a presunti maghi. Plagiano persone di ogni età, istruzione e ceto: stabilire relazioni strette, in particolare quelle amorose, è un bisogno essenziale dell'essere umano. Un utente su tre cerca relazioni online. Sono in tanti quelli che, tra chat e videochiamate, hanno costruito relazioni profonde. Basta sentirsi un po' amati $\mathrm{e}$ la vita cambia. Prezzemolo, salvia, rosmarino e timo $[\ldots]{ }^{11}$

Come in altre prose di Progetto per S. (ad esempio Stinkfist, Poesia dello zenzero, $11 \mathrm{~h}$ (Nuovi modi per uscirne)), anche in questo testo, intitolato Scarborough Fair, è molto difficile identificare un punto di vista enunciativo. La prosa è costruita attraverso un'accumulazione di frasi provenienti dalla televisione, dalla pubblicità o da internet, molte delle quali sono ormai entrate nel linguaggio comune («Basta sentirsi un po' amati e la vita cambia»; oppure, altrove, «Vi va di conoscerla?», «La ricetta per usarlo al meglio»).${ }^{12}$ La nota finale al libro avverte che Scarborough Fair e Poesia dello zenzero sono, in effetti, degli esempi di googlism, dunque si basano

\footnotetext{
${ }^{10} \mathrm{Ci}$ si riferisce alla definizione di poesia derivante dalle poetiche romantiche, intesa cioè come espressione di un contenuto personale da parte di un io che si serve di uno stile soggettivo: cfr. Giuseppe Bernardelli, Il testo lirico. Logica e forma di un tipo letterario, Milano, Vita e Pensiero, 2002 (cfr. soprattutto pp. 27-123); Guido Mazzoni, Sulla poesia moderna, Bologna, il Mulino, 2005 (cfr. soprattutto pp. 43-83). Molti studi novecenteschi hanno ribadito l'opposizione fra poesia e narrazione proprio sulla base del monologismo, a partire da Michael Bachtin, Estetica e romanzo, Torino, Einaudi, 1979, in particolare pp. 67-230. A questo proposito cfr. anche Damiano Frasca, Posture dellio. Luzi, Sereni, Giudici, Caproni, Rosselli, Pisa, Felici, 2014, in particolare pp. 11-19.

${ }^{11}$ Simone Burratti, Progetto per S., Varese, Nuova Editrice Magenta, 2017, p. 45.

${ }^{12}$ ivi, pp. $40-41$.
} 
su un collage di frasi tratte da ricerche su internet. Ciononostante, a differenza di quel che si può dire per altri esempi italiani di googlism, ${ }^{13}$ in questo caso è impossibile non identificare un'intenzionalità autoriale nel testo, che ne condiziona il significato complessivo. Scarborough Fair (il cui titolo e ritornello riprendono una ballata inglese, nonché una canzone di Simon \& Garfunkel) non è una riproduzione meccanica di stralci di ricerche online, bensì un testo che allude a due fenomeni della società occidentale contemporanea: la fiducia nei prodotti che vantano una origine naturale, i quali danno ai consumatori l'illusione di recuperare un rapporto più sano con se stessi - in una sorta di antiscientifico ritorno alla natura -, e la trasformazione dei rapporti interpersonali con il passaggio alla dimensione online. In Scarborough Fair le due tendenze sembrano coincidere; rivelano in modo ironico, ma al tempo stesso drammatico, una esigenza di autenticità e di contatto umano.

La presenza di materiale verbale extraletterario, soprattutto pubblicitario o dei mass media, si ritrova nei testi di molti autori del gruppo di Prosa in prosa, e rimanda a un gesto tipico delle avanguardie; l'uso che ne fa Broggi è interessante perché veicola la prospettiva sul mondo di una voce diversa da quella dell'io. Sia Burratti sia Broggi cercano di rappresentare l'interiorità - riproducibile, plasmata dai media, narcisistica - di soggetti contemporanei. Testi riconducibili a questa tecnica si trovano anche altrove, ad esempio nei libri di Policastro:

11. Sciare in un buco nero (o per meglio dire slittinare)

Lo spettatore-volontario viene munito di un casco e di uno slittino e precipitato in un tubo di plexiglass di dimensioni adattabili alla sala e dalla conformazione a spirale del tipo acquascivolo. La discesa avverrà all'insù, dal pavimento al soffitto che potrebbe, a causa dell'urto, bucarsi. Gradita preparazione atletica (meglio se da stuntmen). Sullo schermo della sala Matthew McConaughey precipita in caduta libera in un buco nero (può agevolare la conoscenza preliminare di Sokal, fornita all'uopo). ${ }^{14}$

Ho avuto molte ragazze, forse perché sono simpatico o per il mio aspetto. Cerco una donna con cui condividere i miei ideali, ovvero il fitness e il divertimento. ${ }^{15}$

È impossibile identificare il soggetto di questa prosa di Policastro, ma è innegabile che non si tratta di un io che coincide con l'autore; inoltre in Esercizi di

\footnotetext{
${ }^{13}$ La presenza di una "terza persona lirica" è stata argomentata anche per esempi italiani di googlism più canonici: al riguardo cfr. Vincenzo Ostuni, Poeti italiani degli anni Zero, Roma, Edizioni Ponte Sisto, 2010, p. 147; e ancora Crocco, La poesia in prosa in Italia, pp. 193-194.

${ }^{14}$ Gilda Policastro, Esercizi di vita pratica, Costa di Rovigo, Prufrock, 2017, p. 26.

${ }^{15}$ Ivi, p. 17.
} 
vita pratica non c’è una unica voce (né, dunque, un unico personaggio). ${ }^{16}$ Lo stesso accade nelle poesie di Carnaroli:

41
papà e mamma
si sono separati
per colpa di mia madre
che si era stufata
delle solite cose
tipo litigate
speriamo che mio padre
un giorno in tribunale
non ci ammazza
con la pistola
o un colpo di pugnale
succedono queste cose
alla televisione
io intanto gli voglio bene
per precauzione

Nei testi considerati fino a ora apparentemente l'autore non ha un proprio punto di vista: sembra venire meno la postura «io autore [ti] sto dicendo che», $i^{18}$ per questo motivo si è parlato di superamento della lirica e della tradizionale assertività del testo poetico. ${ }^{19} \mathrm{Da}$ un punto di vista enunciativo, i soggetti di questi testi - pur

\footnotetext{
${ }^{16}$ Nelle pagine che seguono diamo per scontato che anche in testi lirici è possibile una dimensione di fiction, che rende legittimo parlare di "personaggio": nonostante in alcuni casi la voce sia riconducibile a quella dell'autore, insomma, è inserita in un mondo che non coincide del tutto con quello dell'autore empirico.

${ }^{17}$ Alessandra Carnaroli, Primine, Milano, Edizioni del Verri, 2017, p. 35.

${ }^{18}$ Cfr. Marco Giovenale, Cambio di paradigma, in «il Verri», 43, giugno, pp. 163-165, poi in GAMMM. Anche se la polemica contro l'assertività del testo poetico è stata portata avanti soprattutto da Giovenale, da Inglese e dagli autori vicini a GAMMM, il tentativo di superare l'egocentrismo del gesto lirico proponendone una versione meno monologica precede gli anni Duemila e, come è noto, risale (in Italia) almeno alla poesia degli anni Sessanta: cfr. al riguardo Enrico Testa, Per interposta persona. Lingua e poesia nel secondo Novecento, Roma, Bulzoni, 1999 e Id., Dopo la lirica. Poeti italiani 1960-2000, Torino, Einaudi, 2005; Frasca, Posture dell'io. In tempi più recenti, anche alcuni poeti lirici hanno spiegato di essere alla ricerca di una scrittura in grado di rinunciare ai privilegi dell'io romantico e di esprimere la presenza dell'altro. Cfr. ad esempio Massimo Gezzi, La poetica che si può dire, in «L'Ulisse», 18, 2015, pp. 56-60.

${ }^{19}$ Cfr. ancora Giovenale, Cambio di paradigma; e Marco Giovenale, Corrispondenza privata_(I) assertivo/ non assertivo, in Slowforward. Cfr. anche Gian Luca Picconi, La cornice e il testo. Prammatica della non assertività, Roma, Tic, 2020.
} 
non essendo maschere o doppi dell'io come accadeva nella poesia novecentesca non sono autonomi rispetto all'autore, anche perché nessuno ha uno spazio tale, nella macrostruttura del libro, da potersi definire come personaggio dotato di una propria autocoscienza. ${ }^{20} \mathrm{C}$ 'è una frizione fra le loro parole e la realtà, che dominano sulla superficie, ma non comprendono nelle sue strutture reali: non sono io tormentati né inetti nel senso modernista e novecentesco, bensì figure perfettamente a proprio agio nel lessico e nella sostanza del mondo contemporaneo, le quali in realtà subiscono (e, con misure graduali e variabili di consapevolezza, replicano) le strutture della società capitalista. La consapevolezza di questa frizione genera una forma di straniamento nella percezione del lettore, che non sarebbe presente nel caso di googlism puri o di testi completamente non assertivi. Anche in poesie che si propongono alla lettura come antiliriche, dunque, è presente una forma di soggetto enunciativo (o di voicing), che ne condiziona la lettura e che le separa da analoghi testi non letterari.

Il lessico dei mass media e il montaggio di materiali preesistenti (ma provenienti, in questo caso, soprattutto dal blog dell'autore $)^{21}$ caratterizzano anche i libri di Bortolotti. Tuttavia in Tecniche di basso livello, ma anche in Quando arrivarono gli alieni, c'è una maggiore unità macrotestuale, tanto che si è parlato di dimensione epica. ${ }^{22}$ Inoltre il punto di vista dominante non è quello di figure anonime, bensì di una voce al di sopra delle altre:

Convinti che esistesse una coscienza generata dalla rete, estrapolatasi spontaneamente dai milioni di terabyte di dati, dalle interazioni continue dei server mondiali, in molti iniziarono a sondare i livelli più profondi di internet in cerca di un luogo privilegiato, un punto focale in cui quella coscienza smisurata fosse riuscita a formulare un'individualità. Da qualche parte nel web, sulle piattaforme di social networking, nei circuiti peer-to-peer, la singolarità esprimeva un soggetto e intere comunità on line cercavano di interpellarlo producendo pacchetti di dati e facendoli circolare continuamente, studiando le

\footnotetext{
${ }^{20} \mathrm{Mi}$ servo delle categorie usate da Bachtin, anche se non fa riferimento alla poesia, bensì all'opera di Dostoevskij in opposizione ad altre scritture narrative in prosa: cfr. Michail Bachtin, Dostoevskij. Poetica e stilistica, Torino, Einaudi, 1968, in particolare pp. 64-102.

${ }^{21}$ Si tratta soprattutto del blog "Canopo". Cfr. al riguardo Dimitri Milleri, Gherardo Bortolotti. Montaggio, non finito, critica sociale, in «poesia del nostro tempo», 21 settembre 2020.

${ }^{22}$ Cfr. Paolo Zublena, Politiche del sentirsi in vita. "Tecniche di basso livello" di Gherardo Bortolotti, in «il verri», 46, giugno 2011, pp. 76-81; Chiara De Caprio, Bernardo De Luca, Di 'struttura frasali in cui scarichi le spinte delle tue ragioni. Per un'analisi retorico-stilistica di Senza paragone, in «L'Ulisse», 21, 2018, pp. 302-316.
} 
modifiche che subivano, tentando di ricavare le tracce di un'attività onirica superiore. $^{23}$

In altri testi, trasversalmente a tutta l'opera di Bortolotti, il punto di vista è quello di un "noi":

Gli alieni si stabilirono tra di noi e, intanto, si diffondeva un verbo di prossimità, di secessione. Le periferie si polverizzavano; lungo le tangenziali, i territori si frantumavano sotto la spinta di grandi correnti immaginarie, di epoche psichiche contraddittorie che allontanavano gli amici, i vicini di casa, le rappresentazioni mediatiche. Nei quartieri, per uno strano effetto ottico, persistevano ancora visioni di un futuro comune, di una collettività a venire che ci avrebbe riconosciuto, che ci avrebbe dato ragione, e senza volerlo ne attraversavamo la diafana materia, arrivando all'altro lato, all'altra faccia di qualcosa che non c'era. ${ }^{24}$

A chi si riferisce il «noi»? L'uso connotativo del lessico ${ }^{25}$ indica che in entrambi i casi - sia quando c'è una terza persona, sia quando c’è la prima plurale - in realtà il punto di vista è di un narratore sovrapersonale, che ha più informazioni dei personaggi dei quali si parla e che dunque rielabora i fatti inserendoli in una cornice di senso. ${ }^{26}$ Il linguaggio dei libri di Bortolotti non cerca di imitare quello dei personaggi dei quali si parla (come quello di Broggi), bensì rispecchia una voce che osserva la realtà e riflette su di essa: «le avanguardie della distribuzione al dettaglio», «trame decorose ma banali», «il reale come termine di paragone di qualcosa di cui ti hanno detto, di cui pare sia vero $\gg .{ }^{27} \mathrm{~L}$ 'idea che una conversazione sia senza scopo, che la vita abbia una trama banale o che esista qualcosa di reale diverso dal mondo fisico implica una riflessione di secondo grado sull'esistenza. Anche se la voce delle poesie in prosa di Bortolotti non è tradizionalmente lirica, nel senso di coincidente con quella di un io, su un piano enunciativo propone un punto di vista monologico sulla realtà (in cui i pensieri di più figure anonime sono amalgamate in una unica voce), che ne mette in risalto i punti di contraddizione e di trasformazione per la coscienza moderna. In questo senso, la modalità

\footnotetext{
${ }^{23}$ Gherardo Bortolotti, Quando arrivarono gli alieni. Parti 234-361, Colorno, Tielleci, 2016, pp. 48-49.

${ }^{25}$ Uso il termine "connotativo" nel senso indicato da Gérard Genette, Stile e significato, in Finzione e dizione, Parma, Pratiche, 1994, pp. 77-120, in particolare pp. 80-100. Riguardo al lessico di Bortolotti, cfr. anche Crocco, La poesia in prosa in Italia, pp. 186-187.

${ }^{26} \mathrm{Cfr}$. al riguardo anche Zublena, Politiche del sentirsi in vita, p. 77.

${ }^{27}$ Gherardo Bortolotti, Tecniche di basso livello, Sant'Angelo in Formis, Lavieri, 2009, p. 13; Gherardo Bortolotti, Senza paragone, Massa, Transeuropa, 2013, p. 43; ivi, p. 31.
} 
enunciativa di molti testi di Bortolotti può essere accostata a quella di alcune prose che si trovano nell'ultimo libro di Mazzoni:

Sette anni dopo, a un matrimonio, una vecchia compagna di corso gli parla di un dottorato in Brasile, dei contadini che si inurbano nelle baraccopoli vestiti di stracci, di magliette del Milan, mentre poche decine di chilometri più in là le proprietà terriere superano l'estensione della Svizzera e la classe media si chiude nei quartieri residenziali dietro cancelli altissimi, dietro polizie private. Abita in una zona popolare di Recife, ha un figlio di otto anni; le sembra che le persone vivano per stordirsi nelle feste, che la precarietà sia entrata nella mente e ogni rapporto sia al tempo stesso intimo e superficiale. Lui siede dall'altra parte della tavola nascosto dalle bottiglie, protetto dal proprio volto. Si ripara dalla grandezza del mondo e dai limiti della propria vita fatta di posti in graduatoria, di supplenze alla periferia di Grosseto. Sempre più spesso, quando guarda le persone, le immagina percorrere una traiettoria separata, come se a nessuno importasse più nulla di ciò che non lo tocca personalmente, come se le cose accadessero nella sfera dove le masse si spostano, dove si formano le megalopoli. La sera naviga cercando fotografie di Recife per sentirsi meno inferiore alla vecchia compagna di corso; poi si addormenta, sogna un esame, entra in un'aula piena di alberi e di cani. ${ }^{28}$

Né Mazzoni né Bortolotti si servono del discorso diretto o dell'indiretto libero, ma in entrambi i casi il flusso mentale dei personaggi si interseca con quello dell'autore in un punto di vista intermedio. L'autore ha un livello di conoscenza superiore rispetto a quello dei singoli personaggi, che non appaiono mai in modo autonomo, ma esistono solo nella misura in cui i loro pensieri sono svelati dall'autore. Non si può dire che bgmole - uno dei personaggi dell'opera di Bortolotti - o il protagonista di Recife siano solo lo spettro di un'idea, tuttavia non hanno l'autonomia e lo spazio che potrebbe creare un loro punto di vista sul mondo; sono tasselli di una riflessione più generale - ricostruibile nella macrostruttura del libro e anche dell'opera dell'autore - sull'evoluzione dello spazio intimo e sociale contemporaneo (con una tendenza al racconto distopico, nel caso di Bortolotti; più ibridata con il saggio filosofico, nel caso di Mazzoni).

Mentre i personaggi diversi dall'io in La pura superficie si esauriscono sempre nell'arco di un testo, e non hanno una evoluzione interna, in alcuni libri di Bortolotti ci sono figure ricorrenti (ad esempio in Tecniche di basso livello, ma anche nel recente Storie del pavimento). Una modalità intermedia fra queste due è quella delle voci nei libri di Maccari. Fermate, in particolar modo, è un libro eterogeneo, nel quale si alternano la prima e la terza persona singolare. C’è una focalizzazione

${ }^{28}$ Guido Mazzoni, La pura superficie, Roma, Donzelli, 2017, p. 19. 
interna variabile, con alcuni personaggi che prendono la parola e altri testi in prima persona. Almeno una sezione del libro costituisce una macrosequenza narrativa autonoma: si tratta di Bar, dove le tre poesie Comitiva, Due proprietari e Madre coraggio condividono lo stesso incipit («Nel bar dove vendono la droga») e descrivono un bar di periferia. Il punto di vista è quello di uno dei ragazzi che frequentano il bar e spacciano droga, presentato nella prima poesia:

Nel bar dove vendono la droga

io e i miei amici ci perdiamo

in lunghe briscole tra le bottiglie

che svettano sui tavoli

come riflettori sugli stadi.

Quando i litigi e le zuffe

si fanno quasi violenti

e le pance troppo gonfie di birra

uno di noi si avvicina al bancone

e chiede uno spritz arancione.

Gli viene servito e sotto il tovagliolo

tra piattino e bicchiere si può sfilare

la bustina opaca.

Spesso è presente e connivente

un carabiniere nostra vecchia conoscenza

che sa quel che facciamo e ogni tanto finge,

per farci sorridere, di allarmarsi per il trucco.

Usciamo dal bar e il gruppo

ancora acceso da alterchi sfilacciati

si sposta in una fabbrica in disuso

cento metri vicina.

Uno di noi ha mezzo limone in tasca,

un altro un cucchiaino.

Scendiamo in un'ampia e lurida vasca

di scolo, sediamo, ognuno aspetta il suo turno. ${ }^{29}$

Nei due testi successivi (un poemetto e una poesia in prosa) la voce è ancora quella del ragazzo, ma in realtà vengono rappresentati i mondi di altri personaggi: il barista che deve cedere alle prepotenze di un avventore rabbioso (lo «strano amico»), ${ }^{30}$ l'avventore stesso («il cliente [?] a notte si fece languido| sentimentale,

${ }^{29}$ Paolo Maccari, Fermate, Roma, Elliot, 2017, p. 19.

${ }^{30}$ Ivi, p. 24. 
morbidissimo, | parlò della mamma | di un figlio quasi adolescente | dell'epatite e di rivolta »), ${ }^{31}$ una madre che cerca di allontanare il figlio dalla comitiva degli spacciatori. In alcuni casi le parole sembrano quelle del personaggio secondario, piuttosto che quelle del ragazzo presentato come voce narrante, anche se il passaggio da una voce all'altra non viene segnalato nel testo ( «Meglio accettare uno schiaffo, del resto, da un amico [?] esasperato, fuori di sé, | comunque disperato | e soprattutto sotto di te $\mid$ che prenderle e tacere $\mid$ perché più grosso $\mid$ e fa il muratore $\gg) .32$

In altri punti di Fermate i personaggi vengono introdotti da una voce impersonale e rimangono più anonimi; spesso sono in conflitto con il mondo che li circonda. ${ }^{33}$ A differenza di quanto accade nei testi di Mazzoni e Bortolotti, in quelli di Maccari le figure diverse dall'io non vengono mai chiamate per nome. In tutti e tre i casi, invece, spesso le poesie (e, ancora più di frequente, le poesie in prosa) presentano caratteristiche strutturali tipiche della narrativa: la prospettiva è mutevole e creata con effetti di straniamento, la dimensione temporale è statica o al più reiterativa, ma al centro del testo c'è un evento, per quanto minore o privo di importanza (l'osservazione di un insetto, la passeggiata di un uomo in un parco, una coppia che guarda la televisione); in alcuni casi si fa ricorso al discorso diretto.

Di microracconto si può parlare anche per alcuni testi di Le fuggitive di Carmen Gallo (Torino, Aragno, 2020). Le fuggitive è diviso in tre sezioni: la prima, La corsa, è composta da poesie e prose in cui si distinguono due voci, come segnala l'alternanza tra tondo e corsivo; nella seconda c'è un unico componimento in versi in cui viene usata sempre la prima persona plurale; la terza, Uscirne vivi, è composta $\mathrm{da}$ ventidue prose, più una poesia in versi finale, nelle quali una voce impersonale si alterna a un io per raccontare «storie che hanno a che fare con piccole strategie di lotta e di fuga $\gg .^{34}$ Questi testi hanno un punto di vista simile a quello che abbiamo appena descritto, come si evince da questi esempi:

\section{Certe malattie}

Una mattina la madre di T., affetta da Alzheimer, si è alzata, si è vestita ed è uscita per raggiungere a piedi la casa in cui viveva con suo marito. La famiglia che le ha

\footnotetext{
${ }^{31}$ Ivi, p. 23.

${ }^{32}$ Ivi, pp. 23-24.

${ }^{33}$ La dimensione conflittuale accompagna i personaggi di Maccari fin dai suoi primi libri e in particolare fin da Fuoco amico (Roma, Passigli, 2009), che è anche l'opera con la quale Maccari inizia a esplorare un punto di vista variabile all'interno dei testi. Cfr. al riguardo Marco Villa, Una guerra che non deve finire. Fenomenologia dell'io nella poesia di Paolo Maccari, in «Formavera», 17 novembre 2014.

${ }^{34}$ Carmen Gallo, Le fuggitive, Torino, Aragno, 2020, p. 59.
} 
aperto, notoriamente dedita a traffici illeciti di grande portata, l'ha fatta entrare, le ha preparato il caffe e ha avvisato il figlio che lei era lì e stava bene, e che non c'era fretta di venire a riprendersela. ${ }^{35}$

Rembrandt

Nel museo di Monaco, assediata da altre tele, il Cristo risorto di Rembrandt ritrae il volto di uomo col petto e il torso privo di ferite. Guarito, risanato, mai davvero toccato. Lo sguardo di Cristo fissa bonario e indulgente lo spettatore che non può ricambiare, preso comè a cercare nell'immagine, a scrutare la ferita che manca. $^{36}$

Se Certe malattie ha una struttura narrativa che ricorda alcune prose di Bortolotti, tuttavia, è più difficile analizzare Rembrandt, che, come altre prose dell'ultima sezione (Sealand, La parte migliore, Golden Record) è piuttosto un epigramma che riporta un fatto di cronaca oppure di argomento ecfrastico - un genere che ha poco spazio nella tradizione poetica italiana, per il quale l'unico precedente potrebbe essere considerata l'opera di Giampiero Neri e, in parte, quella di Anedda; ma sono probabili anche modelli non italiani, ad esempio la poesia di Mark Strand.

D'altro tipo è il modo enunciativo delle prose di Jacopo Ramonda, con Una lunghissima rincorsa (Roma, Bel Ami, 2014) e Omonimia (Novara, Interlinea, 2019):

Polo Nord (cut-up n. 21)

Tutte le coppie che conosco si lamentano degli stessi problemi. I mariti, palombari da salotto, affondati nel palinsesto della domenica pomeriggio. I termosifoni in blocco e il Polo Nord in camera da letto, le coperte di calce e i cacciaviti nello stomaco. L'ansia che avvita e svita, che le annoda l'intestino; l'amore che alla fine si rivela un falso d'autore. Lei non parla più, ma non ha segreti: lei è diventata un film muto degli anni Venti. Quando andavamo a scuola, mi raccontavi del rapporto tra i tuoi genitori, come esempio di quello che non volevi; adesso mi parli spesso del tuo primo matrimonio, che è stato un po' come buttarsi sotto un treno, ma poco per volta. ${ }^{37}$

I testi di Ramonda evocano un mondo simile a quello di Broggi, Bortolotti e Mazzoni, fatto di personaggi medi, coppie infelici, frustrazioni, vite alienate. Sono

\footnotetext{
${ }^{35} \mathrm{Ivi}$, p. 38.

${ }^{36}$ Ivi, p. 52.

37 Jacopo Ramonda, Una lunghissima rincorsa. Prose brevi, introduzione di Andrea Inglese, illustrazioni di Ilaria Bossa, Roma, Bel Ami, 2014, p. 83.
} 
prose di lunghezza variabile, talvolta molto lunghe (come nel caso di Black out (cutup n. 149), Strati (cut-up n. 131) e La stasi (cut-up n. 104) in Una lunghissima rincorsa; Nicolo (\#2), Adele (\#1) in Omonimia), che appaiono come microracconti al confine tra poesia in prosa e racconto breve vero e proprio. Spesso sono dedicate a un singolo personaggio, del quale - nella prima sezione del secondo libro prendono il titolo:

Anna (\#2)

Quel poco che ha scoperto, in modo accidentale, le è bastato appena a formulare delle ipotesi, ma è del tutto insufficiente per trarre una qualunque conclusione riguardo al sospetto di essere stata tradita, forse ripetutamente. Anna si chiede cosa le convenga davvero, sapendo di non essere in cerca di risposte che potrebbero aprire ulteriori interrogativi, decisamente più ineludibili. Rivelazioni che la libererebbero dall'inconsapevolezza solo per renderla prigioniera dell'indecisione, costringendola a scegliere tra un numero ristretto di contromosse, per lei tutte egualmente svantaggiose. Poco desiderosa di rivivere il finale del suo primo matrimonio, decide di fermarsi finché è in tempo, determinata - questa volta - a trovare una via d'uscita dentro di sé, forse sopravvalutando il suo senso dell'orientamento e la sua resistenza a stenti e

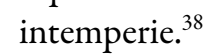

Anche se proprio nel secondo libro i protagonisti dei testi sono dotati di nomi propri, l'idea alla base di Omonimia è la rappresentazione di una interiorità piatta e standardizzata, ${ }^{39}$ che riduce le persone a ombre di pochi modelli imposti dal contesto sociale. Per questo motivo tutte le prose della seconda sezione hanno lo stesso incipit ( «Mi chiamo Andrea »). In questi testi Ramonda usa sempre la prima persona singolare, mentre le prose della prima sezione sono scritte con una terza persona impersonale che ricorda quella dei libri di Bortolotti. In Una lunghissima rincorsa (dove spesso i personaggi sono indicati con una lettera, come a imitare l'iniziale del nome puntata) troviamo sia la prima persona singolare, sia la prima plurale, sia la terza impersonale. Da un punto di vista enunciativo, sono tutti tentativi di rappresentare la voce di soggetti diversi dall'io, e ottengono risultati

\footnotetext{
${ }^{38}$ Jacopo Ramonda, Omonimia, Novara, Interlinea, 2019, p. 23.

${ }^{39}$ Nella Nota al testo si legge che i testi di Omonimia affrontano «il tema dell'identità e dell'omologazione nella società contemporanea, nell'epoca dei social network che, istituendo una sorta di culto della personalità, esaltando e spettacolarizzando la presunta unicità di ogni individuo, come in un talent show della mediocrità, finiscono invece per alimentare il conformismo, l'essere massa, fomentando ancora di più l'illusione di essere unici a cui allude il titolo della raccolta» (Jacopo Ramonda, Nota al testo, in Omonimia, p. 79).
} 
molto simili a quelli di testi pubblicati in collane di narrativa, come Personaggi precari (Rgb, 2007) di Santoni.

Quello che rimane costante nei testi così eterogenei di questo primo gruppo è la fiducia nella capacità della poesia di essere efficace - di affermare una verità, se vogliamo - attraverso figure, rappresentazioni enunciative costruite attraverso tecniche tipiche della fiction. Poco importano il punto di vista di chi parla (di chi sia la voce, appunto), la minore o maggiore verosimiglianza mimetica e l'ironia o il tono tragico. Il bisogno di figure per descrivere stati emotivi - o persino riflessioni sovrapersonali - e la presenza di una voce (o punto di enunciazione) costituiscono una delle caratteristiche intrinseche più interessanti nella poesia contemporanea, che manifesta una continuità (per quanto molti degli autori citati la negherebbero) con l'evoluzione della poesia come genere nel Novecento.

\section{Deflazione ed espansione dell'io}

Questo secondo gruppo si sovrappone al precedente, nel senso che entrambe le tendenze possono essere presenti in uno stesso libro o nell'opera di uno stesso autore. Si tratta dei molti casi in cui l'io è presente, ma in una forma problematica: ad esempio, spesso riflette sulla propria esistenza, mettendo in discussione i propri privilegi; alla diseroicizzazione e all'autoriflessività è speculare, all'opposto, una iper-esposizione del soggetto.

Sul primo versante, vanno considerate innanzitutto le opere di Anedda e di Benedetti. Per Benedetti la scrittura è una sfida allo scorrere del tempo, un modo per provare a far sopravvivere tracce della percezione individuale del mondo, ma è un'impresa destinata al fallimento; molte delle sue poesie sottolineano questa contraddizione. Per sottolineare la parzialità e i limiti di ogni rappresentazione estetica, Benedetti accentua la soggettività del punto di vista, che è volutamente regressivo. ${ }^{40}$ Un'altra strategia è quella di sottolineare il gesto metaletterario: la letteratura è solo una mediazione, e il modo più onesto per vivere la contraddizione epistemologica è esporre la secondarietà del gesto letterario: il libro più rappresentativo di questo secondo atteggiamento è indubbiamente Materiali di un'identità (Massa, Transeuropa, 2010)

Anche Anedda costruisce un soggetto solo apparentemente lirico in senso tradizionale: in realtà spesso è un io che si dissolve in una terza persona impersonale, creando effetti di voicing non lontani da quelli visti nei testi del gruppo

\footnotetext{
${ }^{40}$ Cfr. al riguardo Claudia Crocco, Mario Benedetti da Moriremo guardati (1982) a Il cielo per sempre (1989), in La poesia italiana degli anni Ottanta. Esordi e conferme, a cura di Sabrina Stroppa, Lecce, Pensa Multimedia, 2019, pp. 139-168.
} 
precedente. ${ }^{41}$ Anche quando riprende la prima persona, l'io di Anedda spesso ragiona sulla propria esistenza e sul senso della scrittura, come in questa poesia del suo penultimo libro:

Nuvole, io

I.

Il documento viene salvato, lo schermo torna grigio, lo stesso grigio topo del cielo.

Adesso mi alzerò per sparecchiare.

Vorrei disfarmi dell'io è la moda che prescrive la critica ma la povertà è tale che possiedo solo un pronome.

$\mathrm{Al}$ massimo lo declino al plurale. Dico noi

e mi sento falsamente magnanima.

Dire voi o tu mi dà disagio come accusare.

La terza persona mi confonde ogni volta con il sesso.

Alla fine torno all'io che finge di esistere,

ma è una busta come quelle usate per la spesa

piena di verdura o pesce surgelato.

Io con l'io mi nascondo

chiamando a raccolta quello che sappiamo:

abbiamo paura, ancora non è chiaro come finirà la storia.

Dunque riapro la finestra dello schermo,

ritrovo il documento, esito davanti alla tastiera.

Salvo in una nube l'insalvabile. ${ }^{42}$

Nuvole, io è tratta da Historiae, ma non è l'unico testo di Anedda di questo genere: fa parte di una riflessione sull'identità lirica che si trova in tutta la sua opera. Anche se molte poesie sono autobiografiche, la vita è sempre osservata come dall'esterno, quasi che l'io facesse parte del paesaggio che l'opera di Anedda - nella quale sia la componente visiva sia l'attenzione ai luoghi sono fondamentali $-{ }^{43}$ tenta di rappresentare. Per questo, soprattutto a partire da Dal balcone del corpo (Milano, Mondadori, 2007), spesso la prima persona si disperde in altre voci, che a volte sono antropomorfizzazioni di enti naturali (Parla l'attesa, Parla l'abbandono), a volte si fondono in un coro anonimo (come nei Cori). In Nuvole, io ancora una volta

\footnotetext{
${ }^{41}$ Per una analisi di questo tipo di soggetto nell'opera di Anedda rimando a Crocco, Le poesie italiane di questi anni, e Crocco, La poesia in prosa in Italia, pp. 213-218.

${ }^{42}$ Antonella Anedda, Historiae, Torino, Einaudi, 2018, p. 20.

${ }^{43}$ Cfr. al riguardo Riccardo Donati, Apri gli occhi e resisti. L'opera in versi e in prosa di Antonella Anedda, Roma, Carocci, 2020, soprattutto pp. 38-46.
} 
Anedda rende esplicito un ragionamento sulla questione del soggetto poetico e sul senso della letteratura in generale: l'io «finge di esistere», mostra il gesto poetico nel suo compiersi (come tipico di una tradizione che, nel caso della poesia italiana contemporanea, ha il suo Archetipo in Magrelli di Ora serrata retinae, Milano, Feltrinelli, 1980) e ironizza sulle mode della poesia e della critica (v. 4).

Un caso interessante, fra gli autori più giovani, è Oltre i titoli di coda (Torino, Aragno, 2015) di Giovanna Marmo. Marmo parla soprattutto con la prima persona singolare, ${ }^{44} \mathrm{ma}$ il suo non è senz'altro un io lirico tradizionale. La voce del libro sembra quella di una o più ombre:

Non distinguo nessuno, confondo le persone, anche io voglio essere confusa con gli altri.

Ho perso tutte le informazioni acquisite.

Inutile tracciare l'ombra sul muro

ogni cellula di memoria bruciata

non può essere ricostruita.

Dormo in un film dal montaggio

sempre uguale, con paesaggi di taglio

e scorci in cui non compaiono persone.

Solo sagome, vaghi contorni

in un parcheggio vuoto,

nell'aeroporto dallo spazio trasparente. ${ }^{45}$

Il tema centrale di Oltre $i$ titoli di coda è la visione, ma è sempre deformata: le persone sono solo sagome, e l'io aspira ad essere ridotto a una ombra fra le altre; inoltre ogni scena sembra un frammento isolato dagli altri. Nella sezione centrale (Scomparendo dallo schermo) viene sviluppata la metafora cinematografica che dà il titolo al libro:

Scomparendo dallo schermo

Benvenuti. Accendete la luce ma non guardatemi, perché io non posso riconoscervi, così come voi non siete in grado di riconoscermi.

\footnotetext{
${ }^{44}$ In alcuni casi compaiono anche la prima persona plurale (I primi versi) e la terza singolare (Oltre i titoli di coda, Rumore di unghie, Una cicatrice visiva).

${ }^{45}$ Giovanna Marmo, Oltre i titoli di coda, Torino, Aragno, 2015, p. 26.
} 
Tornate pure al vostro lavoro, rientrate nella cabina di proiezione, niente si modifica.

Ho accettato di essere un duplicato in una stanza composta di carne.

Non indosso nulla, solo una luce nella bocca.

Nonostante il disagio mi stendo sul tavolo di vetro tra tubi al neon.

Respiro dopo respiro

l'aria circonda la mia testa.

È difficile capire chi davvero stia scrivendo.

Vorrei parlare solo in terza persona, attraverso la lingua di cui nessuno può vantare proprietà.

Desidero strofinarmi contro una porta ma non vedo bene, la luce si spegne nella cabina di proiezione. Gli attori entrano in scena a uno a uno mostrando il catalogo dei volti,

ma lei è scomparsa dallo schermo.

Si vede solo la parete alle sue spalle.

$\mathrm{Vi}$ siete mai chiesti se questo è giusto?

Nella stanza di carne il tavolo di vetro è avvolto da un lenzuolo bianco.

Il giorno nasce e muore,

è ora di andare a dormire.

Ancora una volta, arrivederci. ${ }^{46}$

Il libro di Marmo è un catalogo di situazioni private dei dettagli contingenti, nelle quali talvolta si percepisce uno sfondo violento (come nelle poesie Edera ovunque, Rumore di unghie, Unica casa) o l'impossibilità di un contatto autentico fra le persone («Prigionieri in due riprese distinte, | ma speculari, non potevano incontrarsi $\gg),{ }^{47}$ ridotte a fantasmi o comparse. La rarefazione degli eventi reali

${ }^{46}$ Ivi, pp. $27-28$.

${ }^{47}$ Ivi, p. 36. 
( «La trama è composta di fili scuciti, | discorsi riportati, elenchi privi di nessi. | Mi esprimo per quadri e frammenti» $)^{48}$ è una strategia per raccontare la realtà e i rapporti umani: è come se la visione fosse un'ossessione inevitabile («non distogliere l'occhio dall'obiettivo», «è impossibile staccare lo sguardo»), ${ }^{49} \mathrm{ma}$ destinata a essere sfocata, simile a una pellicola bruciata. Di fatto, dunque, l'io che vorrebbe parlare «solo in terza persona», come si legge in Scomparendo dallo schermo, si scinde in frammenti e in voci che non sono realmente soggetti separati, ma piuttosto fantasmi di quella dell'autore.

Una forma di occultamento e di dispersione dell'io c'è anche in Le fuggitive di Gallo. Nella nota finale l'autrice spiega che la sezione centrale (Le fuggitive) è «un'autobiografia per luoghi reali», nella quale «chi parla usa i pronomi per nascondersi $\gg .{ }^{50}$ Questa precisazione, in realtà, potrebbe essere considerata valida per tutto il libro, ${ }^{51}$ e in particolare per le prime due sezioni, dove si alternano prima persona singolare e plurale, terza persona impersonale e discorso diretto:

Siamo in una incubatrice, in una clinica, in un ospedale. Io non sto bene, non respiro bene. Ogni tanto una donna vestita di bianco viene qui e mi prende per i piedi, mi scuote forte. Io non sento niente. Tu sei qui e mi guardi. È tutto in piena luce, ma ci sentiamo al sicuro. ${ }^{52}$

Questa prosa è tratta dalla prima sezione, Una corsa, dedicata al rito greco dell'ephedrismos, un gioco in cui si cerca di rovesciare una pietra colpendola da lontano, e il vincitore deve essere portato sulle spalle dal perdente (bendato) fino a raggiungere la pietra. Nonostante il testo incipitario dia la chiave di lettura della sezione, alla quale si riferiscono alcuni versi, poesie come quella dell'esempio rendono evidente che il tema principale è un altro: tutto il libro è dedicato a incontri conflittuali, a una relazione fra il soggetto principale e un $t u$ ricorrente che ha sempre qualcosa dello scontro. Le figure delle prime due sezioni sono fantasmi,

${ }^{48}$ Ivi, p. 46.

${ }^{49}$ Ivi, p. 38 e p. 44.

${ }^{50}$ Gallo, Le fuggitive, p. 59.

${ }^{51}$ Le fuggitive è un libro più compatto di quanto possa sembrare: ad esempio alcune parole («incubatrice», «bagno», «casa», «stanza») e strutture frasali (ad esempio il «chi» all'inizio di frase, oppure periodi costruiti con "siamo + complemento di luogo", oppure frasi brevi con verbi all'infinito) si ripetono in molti testi; inoltre il lessico rimanda spesso a quella «ossessione visiva», come la definisce Cortellessa nella quarta di copertina, che connota la poesia di Gallo fin dagli esordi, e che la avvicina a quella di Marmo. L'epigrafe proustiana, infine, indica una cornice autobiografica (che non smentisce le varie tecniche di espansione dell'io) che costituisce un ulteriore elemento di coesione del libro.

${ }^{52}$ Gallo, Le fuggitive, p. 11. 
che rendono il soggetto di Le fuggitive sfrangiato e nascosto, come denunciato dall'autrice.

Sul versante dell'iper-esposizione possiamo considerare Mazzoni e Bordini, i quali usano nomi di persona nei propri testi, rendendo evidente l'identità fra autore e voce del testo. Succede in vari punti dei Costruttori di vulcani: «Dieci anni fa il mio amico Beppe Sebaste raccontò a me e a | Giorgio Messori di aver conosciuto un | astrofisico. Questo astrofisico gli disse che il MIT | (Massachusetts Institute of Technology) | invia da diversi anni una serie di messaggi radio nell'universo». ${ }^{53}$ Capita anche nell'ultimo libro di Mazzoni: «Quando Daniele Balicco riprende il controllo siamo vivi, | parliamo di cazzate»; «Telefona a Gigi Simonetti; vuole esserci». ${ }^{54}$ Anche quando usa l'io, si serve di nomi propri e descrive aneddoti che sembrano autobiografici, la poesia di Mazzoni rimane lontana dal pathos che di solito si ascrive alla lirica; la scelta della prima persona è giustificata dall'intenzione poetica più generale di rappresentare il mondo contemporaneo, considerato l'età del narcisismo. ${ }^{55}$ Bordini, invece, parla sempre di sé - parte sempre da una esperienza individuale - e non a caso si serve della categoria di "romanticismo" per descrivere la propria poesia, ${ }^{56} \mathrm{ma}$ decostruendo l'idea che esista un'autenticità dell'io. ${ }^{57}$ Consideriamo una poesia tratta da I costruttori di vulcani:

Questa è una poesia

Se è vero - come ho scritto e come qualcuno mi ha detto che sia vero - che esplorare la propria casa è come esplorare il proprio corpo, allora è vero che esplorare il web è come esplorare la propria anima. Perché ognuno cerca nel web qualcosa che è dentro

la sua anima.

E nella sofferenza delle vittime io vedo la mia sofferenza, e nel loro guardare le loro ferite io vedo me che guardo le mie ferite. La mia ricerca di sofferenza

\footnotetext{
${ }^{53}$ Carlo Bordini, I costruttori di vulcani. Tutte le poesie 1975-2010, Roma, Sossella, 2010, p. 261.

${ }^{54}$ Mazzoni, La pura superficie, p. 76 e p. 63.

${ }^{55}$ Uno dei punti di riferimento della poetica di Mazzoni è Christopher Lasch, La cultura del narcisismo. L'individuo in fuga dal sociale in un'era di disillusioni collettive, Milano, Bompiani, 1999. ${ }^{56} \mathrm{Si}$ vedano le molte interviste nelle quali Bordini parla di romanticismo e «iperverità» a proposito della sua poesia: cfr. ad esempio Carlo Bordini, Autoritratto, a cura di Fabrizio Fantoni, in Poesia. Il blog di Luigia Sorrentino.

${ }^{57}$ Cfr. Gianluigi Simonetti, Su "Difesa berlinese" di Carlo Bordini, in Le parole e le cose 2.
} 
è una ricerca di identità. Sto per comprendere qualcosa di importante di me stesso. Sto per comprendere che il ruolo della vittima mi si confa, e che il mio amore per le vittime è un modo per lenire me stesso, o forse per salvarmi.

Ma se io potessi scegliere, cosa sarei? Sarei una vittima

fiera di esserlo.

Sarei una vittima contenta del

mio ruolo

perdente, del mio sacrificio. (Le parole sono approssimative).

Comprenderei che nella mia vita è sempre stato presente il piacere dell'esibizione.

Se potessi cambierei sesso e sarei una vittima,

col piacere di esibire il mio stato di vittima; $\mathrm{mi}$

sentirei la persona

più importante del mondo per il fatto di avere coraggio e

la franchezza di soffrire. Cercherei l'ammirazione degli altri. Quale immenso orgoglio

si celerebbe dietro alla mia rinuncia a difendermi!

Quale piacere perverso (le parole sono approssimative),

comunque quale piacere, sì, quale piacere,

il piacere di scrivere questa poesia che è più esibizione

che coscienza (quando si è capito veramente qualcosa dirlo è un

atto puramente narcisistico)

"l'enorme secondarietà della letteratura".

$\mathrm{O}$ meglio, questa è una preghiera.

Una cosa mi è stata negata, vivere per gli altri, per far del bene agli altri. Vorrei

vivere senza conflitti, avendo tanti amici, e

ci sono riuscito solo vivendo fuori del mondo.

Ecco, questo è il problema: la bontà. Io trovo della bontà

in queste persone che soffrono. Rinchiudersi in un guscio. Un surrogato della bontà.

(Questa è una poesia sulla bontà).

I siti porno che frequento sono una cosa innocente

Ci sono persone che amano vedere donne che si pisciano sotto ${ }^{58}$

È stato già osservato ${ }^{59}$ che molte poesie di Bordini sembrano il resoconto di sedute psicoanalitiche: sia perché sono costruite associando pensieri a volte molto distanti fra loro in modo caotico, anche da un punto di vista stilistico, sia perché

\footnotetext{
${ }^{58}$ Bordini, I costruttori di vulcani, pp. 294-295.

${ }^{59}$ Ad esempio da Policastro: cfr. Gilda Policastro, Per Carlo Bordini, in Le parole e le cose 2.
} 
ruotano tutte intorno all'interiorità dell'autore. In Questa è una poesia l'autore scrive che «Sto per comprendere | qualcosa di importante di me | stesso», e che «questa è una poesia sulla bontà », ma più avanti si legge che «il piacere di scrivere questa poesia che è più esibizione $\mid$ che coscienza (quando si è capito veramente qualcosa dirlo è un | atto puramente narcisistico)». L'anima viene reificata e ridicolizzata, è qualcosa che può essere esplorato «come il web». Mazzoni e Bordini scrivono poesie molto diverse per stile e per temperatura emotiva, ma entrambi si servono della prima persona singolare senza esitazione né tentativi di mascheramento dell'io; al contrario, in alcuni casi la coincidenza fra autore e soggetto poetico è messa in evidenza all'interno del testo. Entrambi, inoltre, intrecciano l'autobiografia alla saggistica in versi; in questo la riflessione entra a far parte del gesto lirico.

In una zona intermedia tra la deflazione dell'io e la sua iper-esposizione si collocano Ambienti saturi di Donalisio (Venezia, Amos, 2017), ma anche Cronaca senza storia. (Poesie 1999-2015) di Marchesini (Roma, Elliot, 2016), Vita meravigliosa di Cavalli (Torino, Einaudi, 2020) e i testi di esordienti, come Progetto per S. di Burratti, Un paese di soli guardiani di Villa (Venezia, Amos, 2019) e La casa e fuori (Faloppio, Lietocolle, 2019) di Santucci. Sono autori che usano ancora la prima persona, ma in modo meno ingenuo di un tempo, tenendo conto della lezione novecentesca e presentando un io contraddittorio. A proposito di questa modalità enunciativa, rimangono alcune questioni da indagare: ad esempio, quale sia il rapporto fra le posture dell'io appena descritte e quelle di alcuni autori postmontaliani del secondo Novecento; in secondo luogo se sia possibile parlare di autofinzione, per la costruzione dei personaggi autoriali, come da tempo si fa per il romanzo. Questa seconda questione riguarda soprattutto libri nei quali l'identità fra la voce principale del libro e l'io empirico dell'autore è almeno suggerita da dati biografici (come in molti testi di Bordini) o da una somiglianza onomastica (come nel caso di «S.» in Progetto per S. o di «bgmole» nell'opera di Bortolotti). ${ }^{60}$

\section{Poemetto}

La terza tendenza riguarda una tecnica compositiva che influenza anche la voce del testo, ovvero il poemetto. Nella letteratura italiana del Novecento il poemetto è stato associato ai tentativi di raggiungere una forma di polifonia: l'esempio più importante è La ragazza Carla (Milano, Mondadori, 1962) di Elio Pagliarani. Anche nei libri degli anni Duemila ci sono esempi di questo tipo, ma con alcune

${ }^{60}$ Sull'autofinzione in poesia, cfr. Carlo Tirinanzi de Medici, Finzione, discorso, biografia. L'autofiction fra poesia e prosa, in «L'Ulisse», 20, 2017, pp. 6-17. Di doppio autofinzionale, in riferimento a bgmole, si parla anche in Paolo Zublena, Politiche del sentirsi in vita. 
differenze rispetto agli esempi degli anni Sessanta di Pagliarani o di Giorgio Cesarano.

Sono poemetti in cui ci si allontana dal monologismo lirico Ogni cinque bracciate (Firenze, Le Lettere, 2009) di Vincenzo Frungillo, Il sogno di Pasifae di Marco Malvestio (in Roberto Batisti, Francesco Brancati, Marco Malvestio, Hula Apocalisse, Costa di Rovigo, Prufrock 2018) e Uno di nessuno. Storia di Giovanni Antonelli, poeta di Massimo Gezzi (Bellinzona, Casagrande, 2016). Uno di nessuno è la storia di Giovanni Antonelli, poeta marchigiano più volte internato in manicomio. Nella prima parte del libro - cioè il poemetto vero e proprio - il punto di vista è quello di Antonelli e ogni capitolo racconta una parte della sua vita (Infanzia, Mozzo di marina, In carcere, ecc.).

\section{Scrissi parecchie lettere}

a persone ragguardevoli: principi, conti prefetti, sindaci, aguzzini, giudici che mi avete trattato da bandito e disprezzato perché disoccupato, dite, che avreste fatto al posto mio? Umani con chi vi vende una figlia o una sorella, non sapete immaginare il dolore dei vivi.

Eppure siete nudi, iniqui automi: gli orpelli con cui ornate il vostro nulla sono di vetro.

Partii per la Liguria, ossessionato dal delitto.

Ogni giorno ripensavo a Torquato, al suo Tancredi. Mi diedero coraggio, i suoi versi, di notte mi consolarono.

$(\text { Io vivo? io spiro ancora? })^{61}$

La seconda parte è costituita dalla biografia di Antonelli, ricostruita da Gezzi facendo ricorso a testimonianze e documenti d'archivio faticosamente recuperati, nell'intento di «dare un fondamento di verità ai versi che ho scritto». ${ }^{62} \mathrm{~A}$

${ }^{61}$ Massimo Gezzi, Uno di nessuno. Storia di Giovanni Antonelli, poeta, Bellinzona, Casagrande, 2016, p. 21.

${ }^{62}$ Ivi, p. 35. 
proposito dei versi, l'autore avverte che «il personaggio che dice io non è immaginario ma reale, anche se la sua vita, che ha chiesto prepotentemente di essere raccontata in versi, a tratti è immaginata, scorciata, volontariamente travisata ${ }^{63}$ Con Uno di nessuno Gezzi prosegue una ricerca poetica che cerca di sbarazzarsi dei privilegi dell'io lirico tradizionale attraverso una sua democratizzazione: talvolta l'io si disperde in una voce impersonale (come in alcuni testi del libro precedente, $I l$ numero dei vivi), mentre in questo caso diventa una forma di eco per la voce di Antonelli, altrimenti sconosciuta e destinata all'oblio.

Una sperimentazione simile a quella di Gezzi si trova in Il grande innocente (Aragno, 2017) di Gabriel Del Sarto. Nel suo terzo libro Del Sarto include due sezioni che sono, di fatto, due poemetti: Gli uffici e Il grande innocente. Gli uffici è introdotto da una nota in prosa, dove si spiega che il protagonista della sezione è «un uomo di oltre cinquant'anni, il cui nome è Paterson. Egli, raccontando una porzione della sua vita, pone i materiali della sua identità di fronte alle contraddizioni di un'epoca e rivela così la fine di una Storia. Ormai, suggerisce questo narratore, siamo tutti alla deriva $\gg .{ }^{64}$ Nelle righe successive, l'autore spiega l'occasione nella quale ha immaginato questa figura (un progetto di scrittura collettiva del 2014), infine aggiunge che Paterson si basa su persone reali, che ha conosciuto sul lavoro, e sulle loro storie. Il protagonista di Gli uffici, quindi, è un personaggio di finzione che prende la parola in modo diretto; ma la sua dipendenza dal punto di vista dell'autore è rimarcata dal testo stesso. A volte Paterson riporta anche le parole di altri personaggi:

Lungo i giorni dedicati al solo scopo che conosce in questo tempo, anche l'Amministratore Delegato ha avuto paura, è stato uomo.

quando s'imbattono nello stretto $\ll$ Le ore, di un ufficio, con le tapparelle semichiuse e un foglio di calcolo aperto, gelido e pulsante, sono la misura di una carriera - disse e solo la compagnia di una musica sottile è sopportabile». Il ronzio del condizionatore rivela il vuoto degli altri uffici verso sera, il dormiveglia

${ }^{63} \mathrm{Ibid}$.

${ }^{64}$ Gabriel Del Sarto, Il grande innocente, Torino, Aragno, 2017, p. 25. 
dei dati che domani avranno

una vita insensibile e netta. ${ }^{65}$

Nessun altro personaggio di questa sezione, tuttavia, ha lo stesso spazio e lo stesso privilegio di prospettiva di quello principale. Un punto di vista egualmente privilegiato - e distinto da quello dell'autore, anche se dipendente dalla sua esperienza - è solo quello del protagonista dell'altro poemetto, Il grande innocente. Anche in questo caso la sezione è aperta da una nota esplicativa (stavolta in corsivo, e con il titolo di Prologo), dove si legge che il testo è dedicato al nonno dell'autore, ucciso dai tedeschi durante la Resistenza. Come in Gli uffici, anche qui spesso Del Sarto usa la prima persona plurale (alternata alla prima singolare), la quale dà una valenza collettiva al racconto:

Ma questa è la vita, per molti contenuta nell'ora

in cui è possibile, dopo l'ansia

condivisa sul lavoro o nelle cose da fare,

contemplare gli oggetti che abbiamo nel soggiorno di casa,

quell'ora dopo il tramonto in cui potrebbe esserci,

una veranda sul fiume, un frutteto rigonfio

e benedetto da Dio, un silenzio eterno.

La vita è questo, alla fine. Quello che puoi conoscere,

quell'istante convocato dalla sua stesa voce, che riempie e fa il suo silenzio,

che raduna il mondo prima di dissolverlo,

questo e i titoli di coda alla fine del tuo film. Come

una domenica sera, ancora estiva, di questo secolo, infuocata e con un vento

che pulisce la calotta di umidità, la memoria,

vista da un qualsiasi terrazzo di questa collina, proprio sopra la città. ${ }^{66}$

Nel libro di Del Sarto il poemetto amplifica la «diffrazione dell'io» ${ }^{67}$ che caratterizza tutte le sezioni, ma ha anche lo scopo di congiungere la biografia individuale a un vissuto collettivo (la Resistenza storica e quella «mai finita, solo sopita $\gg^{68}$ al capitalismo), dunque di inserire l'io in un contesto meno egocentrico rispetto a quello della lirica tradizionale.

Il libro di poesia italiana recente nel quale il poemetto è anche un modo per realizzare una forma di polifonia è Suite Etnapolis (Novara, Interlinea, 2019) di Lanza. Suite Etnapolis è un libro eterogeneo: composto da versi e da prose, vi si

\footnotetext{
${ }^{65}$ Ivi, p. 29.

${ }^{66}$ Ivi, p. 63.

${ }^{67}$ Così la definisce Raffaele Donnarumma, Recensione a Il grande innocente, in «Allegoria», 78, luglio-dicembre 2018.

${ }^{68}$ Del Sarto, Il grande innocente, p. 60.
} 
alternano narrazione in terza persona e parti dialogiche, ma anche paragrafi che sembrano tratti dalla sceneggiatura di un film o di un documentario (in Sabato) e altri che sembrano riportare messaggi privati (in Giovedi e Venerdi) o un post pubblicato in un social network (ancora in Sabato). Suite Etnapolis è la storia di Etnapolis, un centro commerciale in provincia di Catania, e di alcune persone che vi lavorano. Il lavoro, l'ossessione occidentale per il guadagno («nessun guadagno dopo la scienza | precisa del guadagno, | il lavoro che è pestare i piedi | in un punto solo»), ${ }^{69}$ il precariato e lo sfruttamento dei lavoratori sono senz'altro i temi principali del libro, che Lanza ha scritto «tra il 2013 e il 2015, nel pieno della più spaventosa crisi economica che l'Occidente abbia mai vissuto dal secondo conflitto mondiale»; ${ }^{70}$ eppure Suite Etnapolis non è una riflessione sulle condizioni lavorative della società contemporanea, bensì una forma di satura, nella quale alcuni personaggi raccontano le loro vite, attraverso diverse tecniche espositive, e partecipano a un evento con un chiaro valore metaforico. Laura, Nuccio, Daria, Cinzia, Samuele, Alfredo sono i veri protagonisti del libro: sia perché le loro vicissitudini - tradimenti, relazioni clandestine, violenza domestica, gravidanze, lutti, difficoltà economiche, conflitti e vessazioni lavorative - sono in primo piano nei testi, sia perché prendono la parola anche senza il filtro di un narratore. I monologhi di Suite Etnapolis costruiscono la narrazione, come raramente accade in un libro di poesia, e ancora più raramente nella poesia italiana. Anche l'evento finale del libro, verso il quale tende il racconto generale - la comparsa di un cervo -, è presentato per stralci e punti di vista diversi, e attraverso gli appunti e le interviste prese da un presunto regista che vorrebbe fare un documentario sulla vicenda:

\section{INTERVISTA IV}

Il direttore: Diciamolo subito: il pericolo, come lei dice, c’è. Abbiamo mandato una squadra antincendio a perlustrare i giardini di Etnapolis e il cervo è stato avvistato appena qualche minuto fa, sotto un ulivo, nel settore est del centro commerciale, sul prato di fronte alcuni esercizi di ristorazione, come McDonald. Abbiamo quindi allertato i veterinari dell'ASL di Belpasso, i vigili del fuoco e i carabinieri. Stiamo vietando alla clientela di recarsi negli spazi esterni di Etnapolis, con annunci agli altoparlanti ogni cinque minuti. Ovviamente NON siamo in grado al momento di stabilire come il cervo sia potuto arrivare a Etnapolis, e in generale cosa ci faccia un cervo qui, dal momento che non ci risulta vivano cervi nel catanese. Quello che categoricamente escludo, e che diffido chiunque dal pensare, è che tutto ciò sia una trovata pubblicitaria che qualcuno avrebbe ideato per dare risalto a un centro commerciale come Etnapolis - il primo, lo vorrei ricordare, centro commerciale nato in provincia -

\footnotetext{
${ }^{69}$ Antonio Lanza, Etnapolis, Novara, Interlinea, 2019, p. 69.

${ }^{70}$ Lanza, Nota al testo, in ivi, p. 123.
} 
che di risalto, o ribalta, proprio non ha bisogno. La presenza di un cervo, il pericolo che la presenza di un cervo comporta, faccio notare, non può che creare un danno di immagine, nonché un danno commerciale, per tutta Etnapolis. Altro che trovata pubblicitaria. ${ }^{71}$

\section{INTERVISTA V}

COMMESSA. Anziché andarvene in giro a fare domande del cazzo sulcervodi qua il cervo di là, perché non fate onore alla vostra professione chiedete quali sono le condizioni lavorative di quelli che lavorano qui a Etnapolis, quanti sono in nero, che tipo di contratti hanno, se si sentono rispetati nella loro dignità di lavoratori. Io ad esempio ne ho uno part-time, di contratto, quattro ore per cinque giorni: da venti ore che dovrei fare, sapete quante sono costretta a farne, seno costretta a farne quasi cinquanta a settimana, e sa per quanto, per seicente euro al mese, e non ho diritto alla tredicesima né alle ferie, praticamente diritti non ne ho, solo doveri, e dovrei sentirmi for perché un lavoroce l'ho, un lavoro!, di questo dovreste parlare, di questa specie di colonia penale che chiamano centro commerciale $[\ldots]^{72}$

Le voci frammentarie di Etnapolis contribuiscono a creare una microepica di quel mondo, che cattura l'attenzione del lettore con effetti di suspense (come nella sezione Venerdi, quando l'incidente doloso alla macchina di Laura e l'apparizione del cervo vengono presentati gradualmente, attraverso le chiacchiere di corridoio di Daria a Cinzia, di Cinzia a Vanessa, di Alfredo a Laura ecc.), eventi dal significato metaforico ${ }^{73} \mathrm{e}$ attraverso l'interazione di più personaggi. Il narratore non scompare del tutto: alcuni versi, soprattutto alla fine delle sezioni, sono scritti con la prima persona singolare ( «Dalla terrazza da cui solo mi sporgo $»),{ }^{74}$ come a ricondurre la prospettiva a un io che osserva tutto dall'alto. Rispetto ai casi già visti di Bortolotti, Mazzoni, Maccari ecc., in Etnapolis i personaggi sono più autonomi e compiuti, anche perché si assiste a un loro sviluppo. Oltre alla focalizzazione interna multipla, che presenta un evento contemporaneamente ma da più punti di vista, Lanza si serve di altri espedienti che permettono l'espressione di voci diverse nel discorso principale. Fin dalle prime pagine, ad esempio, la voce dell'altoparlante del centro commerciale si intromette nel discorso del narratore o di un altro personaggio $(\ll$ INVITIAMO TUTTO IL PERSONALE | AD ULTIMARE LE

\footnotetext{
${ }^{71}$ Ivi, pp. 96-97.

${ }^{72}$ Ivi, p. 100.

${ }^{73}$ Ad esempio, la comparsa del cervo: i cervi non vivono in Sicilia, dunque l'evento è impossibile nel mondo reale; il probabile significato metaforico è suggerito anche dall'accenno al mito di Apollo e Ciparisso, tratto dalle Metamorfosi di Ovidio (cfr. ivi, pp. 88-89).

${ }^{74}$ Lanza, Etnapolis, p. 16.
} 
OPERAZIONI | DI APERTURA, GRAZIE») $;^{75}$ in un caso vengono riprodotte le voci dei clienti del centro commerciale. ${ }^{76}$ Solo ai lavoratori, però, vengono dati nomi propri e una caratterizzazione più approfondita: talvolta prendono la parola in modo diretto, come già visto, mentre in altri casi vengono introdotti dal narratore ( $\ll$ in cucina prima di andare, Cinzia [...] studia per strada le carcasse di cani | Ma perché" mormora "nessuno fa niente" $) ; ;^{77}$ infine, in alcuni punti del libro, una didascalia con il loro nome precede quella che sembra la battuta di un copione cinematografico:

\section{SAMUELE:}

Sabato sarò il mio ultimo giorno.

Potrei ripeterlo all'infinito

e non provare niente.

\section{CINZIA:}

Vuoi che non mi dia un piccolo aumento? Da piu di un anno qui, per cinquecento euro al mese. ${ }^{78}$

Anche l'uso del corsivo è molto variabile: a volte segnala la voce di uno dei lavoratori, che però altrove è in tondo; a volte indica una forma di incursione nella loro coscienza:

«Ti vedo... stanca. E non ti prendi cura più di te stessa. Questo, vedo. Sei sicura che con Davide va tutto bene».

«(Mi trova grassa. Ė da mesi che ha smesso di scoparmi. Mi chiama ormai mamma anche lui). Mi vedi preoccupata tu a me? Senti, stacco. Mi preparo, preparo Nicolò, e per le due corro a portartelo a casa tua.$^{79}$

Un altro esempio contemporaneo di poemetto in cui vengono rappresentate voci diverse da quella dell'io si trova nell'opera di Valentino Ronchi. Ronchi ha scritto sia poesia sia narrativa; nella Nota dell'editore posta all'inizio di L'epoca d'oro del cineromanzo (Milano, Nottetempo, 2016) i testi del libro sono definiti «racconti in versi». Fra questi c'è Anna e Mélanie, un poemetto in cui due

\footnotetext{
${ }^{75}$ Ivi, p. 10.

${ }^{76}$ Si tratta della prosa Buste: ivi, pp. 101-103

${ }^{77}$ Ivi, p. 53.

${ }^{78}$ Ivi, p. 23.

${ }^{79}$ Ivi, p. 60.
} 
personaggi femminili vengono introdotti in terza persona nel primo testo; quindi prendono la parola in modo diretto, alternandosi, nelle pagine successive:

\author{
Mio padre ascolta Tutto il calcio \\ minuto per minuto dalla radio \\ nella casa un pomeriggio di settembre \\ l'antenna tirata in lungo e orientata \\ verso il cielo e la finestra \\ s'arrabbia poi gioisce e conserva \\ dei tratti marcati da ragazzo \\ mia madre va spesso da sua madre \\ a chiacchierare sul balcone sottile \\ sopra cortili di fabbriche, io \\ capita vada con loro. Parlano di tempi \\ lontani, appena dietro l'angolo.
}

Per secondo amore un compagno di classe bravo in italiano e in greco e sfrontato con alcune idee vaghe ma certo attraenti su immortalità e bellezza, capelli lunghi quasi al collo e occhi azzurri appena grigi. Un cappuccino insieme alle sette del mattino e lunghi pomeriggi a casa dei suoi, aspettare che la madre esca per baciarsi e sentire la sua mano sotto la gonna ma sopra le calze. ${ }^{80}$

Anche il libro seguente di Ronchi, Primo e parziale resoconto di una storia d'amore, può essere considerato un poemetto. In questo caso il protagonista è un ragazzo innamorato, ed è sua la voce di tutto il libro:

(Presque-rien)

Professore, Hélène sta con me, ci avrà visti. Rientriamo alle nostre case che le famiglie sono già a tavola e mangiamo in fretta con avidità e poi in camera a scriverci lettere per il giorno dopo. Eppure io lo so che potrebbe andar via, o io magari, chissà, che potrebbe finire, lei capisce:

${ }^{80}$ Valentino Ronchi, L'epoca d'oro del cineromanzo. Poesie 2005-2015, Milano, Nottetempo, 2016, pp. 24-25. 
c’è Hélène, ci sono i giorni assieme, ma è come un quasi-niente. Quasi-niente che è molto meglio di niente, potrei dirle, ma lei lo sa di suo - le scrive queste cose - che è così che si vive e s'improvvisa con nulla più che mezze certezze. E altro non si fa che andare avanti e rallentare un attimo talvolta per segnarsi sul quaderno qualche rigo, qualche traccia. ${ }^{81}$

In Primo e parziale resoconto di una storia d'amore si susseguono ambientazioni diverse (Milano, Praga, Lione, Ferrara, Tunisi, Rimini, Roma) e almeno due momenti temporali (alcune estati di fine Novecento e il 1933 della breve sezione Cosa successe a Lione nel 1933. Un nuovo professore). Non c'è un'unica storia d'amore, bensì più innamoramenti: i singoli testi potrebbero essere considerati separatamente. Eppure, nonostante i salti cronologici e spaziali, è proprio la voce del narratore che scoraggia l'ipotesi della lettura separata delle poesie. Il punto di vista del ragazzo innamorato, al quale non viene mai dato un nome, è ciò che dà compattezza al libro: la voce non si limita a raccontare o a esporre i propri pensieri, ma costruisce un mondo finzionale.

\section{Ibridi}

Un'altra tendenza della poesia italiana contemporanea è quella a essere sempre più installativa, cioè a mescidarsi con altre forme artistiche, soprattutto visuali: è quel che accade, ad esempio, in Nel gasometro di Sara Ventroni (Firenze, Le Lettere, 2006) e nel successivo La sommersione (Torino, Aragno, 2016). La poesia installativa è quella che ha bisogno di un ruolo attivo del lettore (che diventa anche performer o prosumer) ${ }^{82}$ per essere riconosciuta; molto spesso questa categoria è stata usata in riferimento ad autori che scrivono con un linguaggio che è nell'orizzonte neoavanguardistico, ad esempio con forme di asintattismo e di montaggio. In un senso più generale, possiamo considerare in questa quarta tendenza anche libri la cui sperimentazione si gioca su un piano diverso, apparentemente più tradizionale, ma la cui struttura si basa su una espansione quasi uno sconfinamento - in altri generi artistici, che integrano il testo poetico e ne completano il significato: ad esempio opere ibride come I camminatori di Italo Testa (Livorno, Valigie rosse, 2013), i Faldoni di Vincenzo Ostuni ${ }^{83}$ e Salva con nome (Milano, Mondadori, 2012) di Anedda.

${ }^{81}$ Id., Primo e parziale resoconto di una storia d'amore, Milano, Nottetempo, 2017, p. 30.

${ }^{82}$ Giovannetti, La poesia italiana degli anni Duemila, pp. 37-58.

${ }^{83}$ Le varie versioni del Faldone di Vincenzo Ostuni sono state pubblicate presso Oèdipus, Ponte Sisto, Aragno e Tic, come si legge sul sito Faldone. 


\section{Prosa}

Alcuni libri di poesia nominati in queste pagine includono anche prose (ad esempio Progetto per S., Fermate, Suite Etnapolis) o sono scritti interamente in prosa (Tecniche di basso livello, Una lunghissima rincorsa). Ciò non stupisce, in quanto la poesia in prosa è una forma letteraria che acquisisce autonomia estetica nei periodi di ridefinizione dei generi e, come si è mostrato, negli ultimi due decenni sono state tentate molte strade per allontanare la poesia italiana dal modello lirico tradizionale. La poesia in prosa fa parte di questi tentativi. Dopo essere comparsa solo in modo episodico per gran parte del secondo Novecento - fatta eccezione per l'opera di Giampiero Neri - tra la fine del secolo e l'inizio del successivo alcune sperimentazioni di poesia senza verso sono avvenute quasi contemporaneamente da parte di poeti molto diversi l'uno dall'altro (ad esempio Magrelli, quindi Dal Bianco, Anedda, Mazzoni, Inglese, Raos, Benedetti, Bortolotti); tuttavia negli anni Dieci la poesia in prosa è stata identificata soprattutto con quella di un gruppo di autori riuniti in alcuni blog (GAMMM) e in una antologia (Prosa in prosa, Firenze, Le Lettere, 2009). Prosa in prosa ha contribuito a evidenziare i punti di saturazione e di usura della poesia considerata egemone fino a quel momento, cioè la lirica. Questo gruppo ha assunto un atteggiamento militante, comportandosi come una avanguardia, con interventi critici sulle riviste online, attraverso le traduzioni e le pubblicazioni, ma anche organizzando eventi letterari e, infine, servendosi dei social network. Di conseguenza sono passati in secondo piano altri tipi di poesia in prosa, altrettanto meritevoli di interesse, che provengono da esperienze e modelli diversi rispetto a quelli di GAMMM e Prosa in prosa. ${ }^{84}$

\section{Conclusione}

Le poesie italiane di questi anni esplorano i limiti dello spazio letterario ${ }^{85}$ della poesia. Dall'assenza del verso alla ricerca di un punto di vista enunciativo che rispecchi una soggettività diversa dell'autore, passando per il depotenziamento o la scissione dell'io e lo sconfinamento in altri generi artistici, le tendenze appena esaminate mostrano che la poesia contemporanea italiana cerca sempre più di allontanarsi dal modello romantico e poi novecentesco basato sul monologismo e sulla prevalenza del verso. La poesia, in un certo senso, tenta di uscire da sé stessa. Eppure qualcosa rimane costante: il tentativo di rappresentare forme di interiorità, ricorrendo a immagini - e, in misura variabile, a tecniche narrative - e richiedendo

\footnotetext{
${ }^{84} \mathrm{Cfr}$. al riguardo, anche per la definizione di poesia in prosa, Crocco, La poesia in prosa in Italia, soprattutto pp. 11-15 e 231-235.

${ }^{85}$ Uso questa espressione nel senso delineato da Guido Mazzoni, Sulla poesia moderna, Bologna, il Mulino, 2005, p. 9.
} 
al lettore di accettare la validità epistemologica del testo indipendentemente dall'identità del punto di vista enunciativo, ma in virtù della sua presenza. In questo senso, i testi poetici sembrano stabilire un patto con il lettore la cui natura è ancora da chiarire.

\section{Bibliografia}

Andrea Afribo, Deangelisiana, in Poesia '70-'80: le nuove generazioni. Geografia e storia, opere e percorsi, letture e commento, selezione di contenuti dal convegno (Torino, 15-17 dicembre 2015), a cura di Davide Dalmas, Stefano Giovannuzzi, Beatrice Manetti e Sabrina Stroppa, Genova, San Marco dei Giustiniani, pp. 113-132.

Giancarlo Alfano, Alessandro Baldacci, Cecilia Bello Minciacchi, Andrea Cortellessa, Massimiliano Manganelli, Raffaella Scarpa, Fabio Zinelli, Paolo Zublena, Parola plurale. Sessantaquattro poeti fra due secoli, Roma, Sossella, 2005.

Antonella Anedda, Il catalogo della gioia, Roma, Donzelli, 2003.

Antonella Anedda, Dal balcone del corpo, Milano, Mondadori, 2007.

Antonella Anedda, Salva con nome, Milano, Mondadori, 2012.

Antonella Anedda, Historiae, Torino, Einaudi, 2018.

Antonella Anedda, Geografie, Milano, Garzanti, 2021.

Michail Bachtin, Dostoevskij. Poetica e stilistica, Torino, Einaudi, 1968.

Michail Bachtin, Estetica e romanzo, Torino, Einaudi, 1979.

Mario Benedetti, Materiali di un'identità, Massa, Transeuropa, 2010.

Alfonso Berardinelli, Franco Cordelli, a cura di, Il pubblico della poesia, Cosenza, Lerici, 1975.

Giuseppe Bernardelli, Il testo lirico. Logica e forma di un tipo letterario, Vita e Pensiero, Milano, 2002.

Carlo Bordini, I costruttori di vulcani. Tutte le poesie 1975-2010, Roma, Sossella, 2010.

Gherardo Bortolotti, Tecniche di basso livello, Sant'Angelo in Formis, Lavieri, 2009.

Gherardo Bortolotti, Senza paragone, Massa, Transeuropa, 2013. 
Gherardo Bortolotti, Oltre il pubblico: la letteratura e il passaggio alla rete, in Idee della prosa, a cura di Gilda Policastro, numero monografico di «Nuova prosa», 6, 2014, pp. 77-146, poi pubblicato oline su Leparole e le cose, 22 dicembre 2014, http://www.leparoleelecose.it/?p=17196.

Gherardo Bortolotti, Quando arrivarono gli alieni. Parti 234-361, Colorno, Tielleci, 2016.

Gherardo Bortolotti, Low, Roma, Tic, 2020.

Alessandro Broggi, Avventure minime, Massa, Transeuropa, 2014.

Alessandro Broggi, Noi, Roma, Tic, 2021.

Simone Burratti, Progetto per S., Varese, Nuova Editrice Magenta, 2017.

Alessandra Carnaroli, Primine, Milano, Edizioni del Verri, 2017.

Patrizia Cavalli, Vita meravigliosa, Torino, Einaudi, 2020.

Claudia Crocco, Mario Benedetti da Moriremo guardati (1982) a Il cielo per sempre (1989), in La poesia italiana degli anni Ottanta. Esordi e conferme, a cura di Sabrina Stroppa, Lecce, Pensa Multimedia, 2019, pp. 139-168.

Claudia Crocco, La poesia in prosa in Italia. Dal Novecento a oggi, Roma, Carocci, 2021.

Jonathan Culler, Theory of the Lyric, Cambridge, Harvard University Press, 2015.

Chiara De Caprio, Bernardo De Luca, Di 'strutture frasali in cui scarichi le spinte delle tue ragioni'. Per un'analisi retorico-stilistica di Senza paragone, in «L'Ulisse», 21, 2018, pp. 302-316.

$<$ https://www.lietocolle.com/2018/10/ulisse-n-21-anno-2018-saggi-in-versi-saggi-poeticilyrical-essays-forme-ibride-e-innesti-nelle-scritture-contemporanee/>

Gabriel Del Sarto, Il grande innocente, Torino, Aragno, 2017.

Fabio Donalisio, Ambienti saturi, Venezia, Amos, 2017.

Riccardo Donati, Apri gli occhi e resisti. L'opera in versi e in prosa di Antonella Anedda, Roma, Carocci, 2020.

Raffaele Donnarumma, Recensione a Il grande innocente, in «Allegoria», 78, luglio-dicembre 2018.

$<$ https://www.allegoriaonline.it/index.php/i-numeri-precedenti/allegoria-n-78/149-tremilabattute/78/1127-gabriel-del-sarto-il-grande-innocente>

Gian Carlo Ferretti, Giulia Iannuzzi, Storie di uomini e libri. L'editoria italiana attraverso le sue collane, Roma, minimum fax, 2014. 
Franco Fortini, Saggi ed epigrammi, a cura e con un saggio introduttivo di Luca Lenzini e uno scritto di Rossana Rossanda, Milano, Mondadori, 2003

Damiano Frasca, Posture dellio. Luzi, Sereni, Giudici, Caproni, Rosselli, Pisa, Felici, 2014.

Vincenzo Frungillo, Ogni cinque bracciate, Firenze, Le Lettere, 2009.

Carmen Gallo, Le fuggitive, Torino, Aragno, 2020.

Gérard Genette, Finzione e dizione, Parma, Pratiche, 1994.

Massimo Gezzi, Uno di nessuno. Storia di Giovanni Antonelli, poeta, Bellinzona, Casagrande, 2016.

Massimo Gezzi, La poetica che si può dire, in «L'Ulisse», 18, 2015, pp. 56-60. <http://www.lietocolle.com/cms/wp-content/uploads/2014/03/ULISSE-182.pdf>

Paolo Giovannetti, La poesia italiana degli anni Duemila. Un percorso di lettura, Roma, Carocci, 2017.

Marco Giovenale, Cambio di paradigma, in «il Verri», 43, giugno, pp. 163-165, poi in GAMMM, 10 febbraio 2011.

<https://gammm.org/2011/02/10/cambio-di-paradigma/>

Francesco Guglieri, Michele Sisto, Verifica dei poteri 2.0. Critica e militanza letteraria in internet (1999-2009), in «Allegoria», 61, 2010, pp. 153-174.

Käte Hamburger, La logica della letteratura, a cura di Eleonora Caramelli, Bologna, Pendragon, 2015.

Antonio Lanza, Etnapolis, Novara, Interlinea, 2019.

Christopher Lasch, La cultura del narcisismo. L'individuo in fuga dal sociale in un'era di disillusioni collettive, Milano, Bompiani, 1999.

Paolo Maccari, Fuoco amico, Roma, Passigli, 2009.

Paolo Maccari, Fermate, Roma, Elliot, 2017.

Paolo Maccari, Iferri corti, Faloppio, Lietocolle, 2020.

Valerio Magrelli, Ora serrata retinae, Milano, Feltrinelli, 1980.

Marco Malvestio, Il sogno di Pasifae, in Roberto Batisti, Francesco Brancati, Marco Malvestio, Hula Apocalisse, Costa di Rovigo, Prufrock, 2018.

Matteo Marchesini, Cronaca senza storia. (Poesie 1999-2015), Roma, Elliot, 2016.

Giovanna Marmo, Oltre i titoli di coda, Torino, Aragno, 2015. 
Guido Mazzoni, Sulla poesia moderna, Bologna, il Mulino, 2005.

Guido Mazzoni, I mondi, Roma, Donzelli, 2010

Guido Mazzoni, La pura superficie, Roma, Donzelli, 2017.

Guido Mazzoni, Sulla storia sociale della poesia contemporanea in Italia, in «Ticontre. Teoria Testo Traduzione», VIII, 2017, pp. 1-26. $<$ http://www.ticontre.org/ojs/index.php/t3/article/view/236/179>

Vincenzo Ostuni, Poeti italiani degli anni Zero Roma, Edizioni Ponte Sisto, 2010.

Elio Pagliarani, La ragazza Carla e altre poesie, Milano, Mondadori, 1962.

Gian Luca Picconi, La cornice e il testo. Prammatica della non assertività, Roma, Tic, 2020.

Gilda Policastro, Esercizi di vita pratica, Costa di Rovigo, Prufrock, 2017.

Jacopo Ramonda, Una lunghissima rincorsa. Prose brevi, introduzione di Andrea Inglese, illustrazioni di Ilaria Bossa, Roma, Bel Ami, 2014.

Jacopo Ramonda, Omonimia, Novara, Interlinea, 2019.

Valentino Ronchi, L'epoca d'oro del cineromanzo. Poesie 2005-2015, Roma, Nottetempo, 2016.

Valentino Ronchi, Primo e parziale resoconto di una storia d'amore, Roma, Nottetempo, 2017.

Francesca Sante, Design Thinking: studio preparatorio per un progetto in versi, tesi di laurea, Corso di Laurea in Economia e Gestione delle Arti e Attività culturali, a.a. 2017/2018, Università Ca' Foscari Venezia, relatore prof. Monica Calcagno, controrelatore prof. Ricciarda Ricorda.

Vanni Santoni, Personaggi precari, Rgb, 2007, poi con postfazione di Raoul Bruni, Roma, Voland, 2013.

Francesca Santucci, La casa e fuori, Faloppio, Lietocolle, 2019.

Damiano Sinfonico, Scuola deangelisiana: l'esempio della collana Niebo, in «Ticontre. Teoria Testo Traduzione», VIII, 2017, pp. 73-85. http://www.ticontre.org/ojs/index.php/t3/article/view/186/195.

Enrico Testa, Per interposta persona. Lingua e poesia nel secondo Novecento, Roma, Bulzoni, 1999.

Enrico Testa, Dopo la lirica. Poeti italiani 1960-2000, Torino, Einaudi, 2005.

Italo Testa, I camminatori, Livorno, Valigie rosse, 2013. 
Carlo Tirinanzi de Medici, Finzione, discorso, biografia. L'autofiction fra poesia e prosa, in «L'Ulisse», 20, 2017, pp. 6-17.

$<$ https://www.lietocolle.com/cms/wp-content/uploads/2017/07/U20-Poesia-autofiction-ebiografia-1.pdf>

Sara Ventroni, Nel gasometro, Firenze, Le Lettere, 2006.

Sara Ventroni, La sommersione, Torino, Aragno, 2016.

Marco Villa, Un paese di soli guardiani, Venezia, Amos, 2019.

Paolo Zublena, Politiche del sentirsi in vita. "Tecniche di basso livello" di Gherardo Bortolotti, in «il verri», 46, giugno 2011, pp. 76-81.

\section{Sitografia}

Carlo Bordini, Autoritratto, a cura di Fabrizio Fantoni, in Poesia. Il blog di Luigia Sorrentino, 17 dicembre 2015.

<http://poesia.blog.rainews.it/2015/12/carlo-bordini/>

Marco Giovenale, Corrispondenza privata_(I) assertivo/ non assertivo, in «Slowforward», 5 luglio 2013.

$<$ https://slowforward.net/2013/07/05/corrispondenza-privata-_-1-assertivo-nonassertivo/>

Andrea Lombardi, L'esperienza di «Nazione indiana» nella storia del web letterario italiano, in «L'Ulisse», 19, 2016, pp. 47-63.

<http://www.lietocolle.com/cms/wp-content/uploads/2016/04/ULISSE-19.pdf>

Dimitri Milleri, Gherardo Bortolotti. Montaggio, non finito, critica sociale, in Poesia del nostro tempo, 21 settembre 2020.

$<$ https://www.poesiadelnostrotempo.it/gherardo-bortolotti-installazioni-non-finito-criticasociale/>

Vincenzo Ostuni, Faldone.

$<$ http://www.faldone.it/>

Gilda Policastro, Per Carlo Bordini, in Le parole e le cose 2, 10 novembre 2020. $<$ http://www.leparoleelecose.it/?p=39715>

Gianluigi Simonetti, Su "Difesa berlinese" di Carlo Bordini, in Le parole e le cose 2, 7 aprile 2019.

$<$ http://www.leparoleelecose.it/?p=35304>

Marco Villa, Una guerra che non deve finire. Fenomenologia dell'io nella poesia di Paolo Maccari, in Formavera, 17 novembre 2014.

$<$ https://formavera.com/2014/11/17/una-guerra-che-non-deve-finire-fenomenologia-dellionella-poesia-di-paolo-maccari-2/> 